\title{
Sharp Embeddings of Besov Spaces with Logarithmic Smoothness
}

\author{
Petr GURKA and Bohumír OPIC
}

\author{
Department of Mathematics \\ Czech University of Agriculture \\ 16521 Prague 6, Czech Republic \\ gurka@tf.czu.cz
}

\author{
Mathematical Institute \\ Academy of Sciences of the Czech Republic \\ Žitná 25, 11567 Prague 1, Czech Republic \\ opic@math.cas.cz
}

Recibido: 26 de Abril de 2004

Aceptado: 17 de Junio de 2004

\begin{abstract}
We prove sharp embeddings of Besov spaces $B_{p, r}^{\sigma, \alpha}\left(\mathbb{R}^{n}\right)$ with the classical smoothness $\sigma$ and a logarithmic smoothness $\alpha$ into Lorentz-Zygmund spaces. Our results extend those with $\alpha=0$, which have been proved by D. E. Edmunds and H. Triebel. On page 88 of their paper (Math. Nachr. 207 (1999), 79-92) they have written: "Nevertheless a direct proof, avoiding the machinery of function spaces, would be desirable." In our paper we give such a proof even in a more general context. We cover both the sub-limiting and the limiting cases and we determine growth envelopes of Besov spaces with logarithmic smoothness.
\end{abstract}

Key words: Besov spaces with logarithmic smoothness, Lorentz-Zygmund spaces, sharp embeddings.

2000 Mathematics Subject Classification: 46E35, 46E30, 26D10.

\section{Introduction}

The aim of this paper is to prove sharp embeddings of Besov spaces $B_{p, r}^{\sigma, \alpha}\left(\mathbb{R}^{n}\right)$ with the classical smoothness $\sigma$ and a logarithmic smoothness $\alpha$ into Lorentz-Zygmund spaces both in sub-limiting and limiting cases. As mentioned above, our results extend the corresponding ones from $[15,22,23]$, where $\alpha=0$. Our methods, based on those of $[9,12,20]$, are quite elementary and can be extended to the case of Besov spaces of

The research was partially supported by grant no. 201/01/0333 of the Grant Agency of the Czech Republic and by the Friedrich Schiller University in Jena. 
generalized smoothness. In contrast to $[15,22]$, we do not use the interpolation theory (to prove embeddings) and the atomic decomposition of spaces $B_{p, r}^{\sigma, \alpha}\left(\mathbb{R}^{n}\right)$ (to prove the sharpness of embeddings). We also establish growth envelopes of spaces in question. For basic facts about this notion we refer to [16,23]. Note also that growth envelopes of Besov spaces with generalized smoothness are established in $[5,6]$. However, the authors of these papers again make use of atomic decompositions to get their results.

Our paper is organized as follows. In section 1 we introduce the notation and give basic definitions. The main results are presented in section 2 while their proofs are given in sections 3 and 4 . The proofs of some facts concerning growth envelopes can be found in the Appendix.

\section{Notation and preliminaries}

We write $A \lesssim B$ (or $A \gtrsim B$ ) if $A \leq c B$ (or $c A \geq B$ ) for some positive constant $c$ independent of appropriate quantities involved in the expressions $A$ and $B$, and $A \approx B$ if $A \lesssim B$ and $A \gtrsim B$. For $p \in[1, \infty]$, the conjugate number $p^{\prime}$ is defined by $1 / p+1 / p^{\prime}=1$ with the convention that $1 / \infty=0$.

Let $\Omega$ be a measurable subset of $\mathbb{R}^{n}$ (with respect to $n$-dimensional Lebesgue measure); by $|\Omega|_{n}$ we mean its $(n)$-volume while $\chi_{\Omega}$ stands for the characteristic function of $\Omega$. The volume and the surface area of the unit ball $B_{n}(0,1)$ in $\mathbb{R}^{n}$ is denoted by $\beta_{n}$ and by $s_{n}$, respectively. The symbol $\mathfrak{M}(\Omega)$ is used to denote the family of all scalar-valued (real or complex) measurable functions on the set $\Omega$. By $\mathfrak{M}^{+}(\Omega)$ we mean the subset of $\mathfrak{M}(\Omega)$ consisting of those functions which are non-negative a.e. on $\Omega$. If $\Omega=(a, b) \subseteq \mathbb{R}$, we write simply $\mathfrak{M}(a, b)$ and $\mathfrak{M}^{+}(a, b)$ instead of $\mathfrak{M}((a, b))$ and $\mathfrak{M}^{+}((a, b))$. Finally, $\mathfrak{M}^{+}(a, b ; \downarrow)$ stands for the collection of all $f \in \mathfrak{M}^{+}(a, b)$ which are non-increasing on $(a, b)$.

\subsection{Embeddings}

Given two (quasi-)Banach spaces $X$ and $Y$, we write $X=Y$ (and say that $X$ and $Y$ coincide) if $X$ and $Y$ are equal in the algebraic and the topological sense (their (quasi-)norms are equivalent). The symbol $X \hookrightarrow Y$ means that $X \subset Y$ and the natural embedding of $X$ in $Y$ is continuous.

\subsection{Generalized Lorentz-Zygmund spaces}

Let $p, q \in(0, \infty], m \in \mathbb{N}, \alpha_{1}, \ldots, \alpha_{m} \in \mathbb{R}$ and let $\Omega$ be a measurable subset of $\mathbb{R}^{n}$. The generalized Lorentz-Zygmund (GLZ) space $L_{p, q ; \alpha_{1}, \ldots, \alpha_{m}}(\Omega)$ consists of all functions $f \in \mathfrak{M}(\Omega)$ such that the quantity

$$
\|f\|_{p, q ; \alpha_{1}, \ldots, \alpha_{m} ; \Omega}=\left\|t^{1 / p-1 / q}\left(\prod_{j=1}^{m} \ell_{j}^{\alpha_{j}}(t)\right) f^{*}(t)\right\|_{q ;(0, \infty)}
$$


is finite, where $\ell_{1}, \ldots, \ell_{m}$ are (logarithmic) functions defined on $(0, \infty)$ by

$$
\ell_{1}(t)=\ell(t)=1+|\log t|, \quad \ell_{j}(t)=\ell\left(\ell_{j-1}\right)(t) \quad(j>1),
$$

$f^{*}$ denotes the non-increasing rearrangement of $f$ given by

$$
f^{*}(t)=\inf \left\{\lambda>0:|\{x \in \Omega:|f(x)|>\lambda\}|_{n} \leq t\right\}, \quad t \geq 0,
$$

and $\|\cdot\|_{q ; E}$ is the usual $L^{q}$-(quasi-)norm on the set $E$.

We shall also need the maximal function $f^{* *}$ of $f^{*}$ defined by

$$
f^{* *}(t)=\frac{1}{t} \int_{0}^{t} f^{*}(s) \mathrm{d} s, \quad t>0
$$

(clearly, $\left.f^{*} \leq f^{* *}\right)$.

Let us note that when each $\alpha_{j}=0$, the space $L_{p, q ; \alpha_{1}, \ldots, \alpha_{m}}(\Omega)$ coincides with the Lorentz space $L^{p, q}(\Omega)$ which is just the Lebesgue space $L^{p}(\Omega)$ when $p=q$. If $m=1$, $L_{p, q ; \alpha_{1}}(\Omega)$ is the Lorentz-Zygmund space $L^{p, q}(\log L)^{\alpha_{1}}(\Omega)$ introduced in [2] which, when $p=q$, is the Zygmund class $L^{p}(\log L)^{\alpha_{1}}(\Omega)$. The spaces $L_{p, q ; \alpha_{1}, \ldots, \alpha_{m}}(\Omega)$ were studied in $[7-12,19]$, where more information can be found.

If $\Omega=\mathbb{R}^{n}$, we sometimes omit this symbol in the notation and, for example, simply write $\|\cdot\|_{p, q ; \alpha_{1}, \ldots, \alpha_{m}}$ or $L_{p, q ; \alpha_{1}, \ldots, \alpha_{m}}$ instead of $\|\cdot\|_{p, q ; \alpha_{1}, \ldots, \alpha_{m} ; \mathbb{R}^{n}}$ or $L_{p, q ; \alpha_{1}, \ldots, \alpha_{m}}\left(\mathbb{R}^{n}\right)$, respectively.

\subsection{Orlicz spaces}

Let $\Phi$ be a Young function (that is, a continuous, non-negative, strictly increasing, convex function on $[0, \infty)$ such that $\left.\lim _{t \rightarrow 0_{+}} \Phi(t) / t=\lim _{t \rightarrow \infty} t / \Phi(t)=0\right)$ and let $\Omega$ be a measurable subset of $\mathbb{R}^{n}$. By $L_{\Phi}(\Omega)$ we denote the corresponding Orlicz space, equipped with the Luxemburg norm $\|\cdot\|_{\Phi ; \Omega}$ (for details of such spaces we refer to $[1,3,17])$. Orlicz spaces and GLZ spaces are two different classes of function spaces having a nontrivial intersection. For example (see $\left[2\right.$, Thm. D]), if $\Omega$ is a domain in $\mathbb{R}^{n}$ with $|\Omega|_{n}<\infty$ and $\nu>0$, then the space $L_{\infty, \infty ;-1 / \nu}(\Omega)$ coincides with the Orlicz space $L_{\Phi}(\Omega)$, whose Young function satisfies $\Phi(t) \approx \exp t^{\nu}$ for all large $t$. Thus, in this case we put

$$
\operatorname{EXP} L^{\nu}(\Omega)=L_{\infty, \infty ;-1 / \nu}(\Omega)
$$

\subsection{Besov spaces with logarithmic smoothness}

For each $h \in \mathbb{R}^{n}$ the first difference operator, $\Delta_{h} \equiv \Delta_{h}^{1}$, is defined on functions on $\mathbb{R}^{n}$ by

$$
\Delta_{h} f(x)=f(x+h)-f(x), \quad x \in \mathbb{R}^{n},
$$

and higher order differences of $f$ are defined inductively by

$$
\Delta_{h}^{k+1} f(x)=\Delta_{h}\left(\Delta_{h}^{k} f\right), \quad k \in \mathbb{N} .
$$


The $k$-th order modulus of continuity of a function $f \in L^{p}\left(\mathbb{R}^{n}\right), 1 \leq p<\infty$, or $f \in L^{\infty}\left(\mathbb{R}^{n}\right) \cap C\left(\mathbb{R}^{n}\right)$, is given by

$$
\omega_{k}(f, t)_{p}=\sup _{|h| \leq t}\left\|\Delta_{h}^{k} f\right\|_{p} \quad \text { for all } t>0 .
$$

Each modulus $\omega_{k}(f, t)_{p}, 1 \leq p \leq \infty$, is a non-negative non-decreasing function of $t>0$. Furthermore,

$$
\omega_{k}(f, t)_{p} \leq 2^{k}\|f\|_{p} .
$$

Let $1 \leq p, r \leq \infty, \sigma>0$ and $\alpha \in \mathbb{R}$. Let $M$ be a positive integer such that $M>\sigma$. The Besov space $B_{p, r}^{\sigma, \alpha}\left(\mathbb{R}^{n}\right)$ (with the classical smoothness $\sigma$ and the logarithmic smoothness $\alpha$ ) consists of those functions $f \in L^{p}\left(\mathbb{R}^{n}\right)$ (if $\left.p<\infty\right)$ or $f \in L^{\infty}\left(\mathbb{R}^{n}\right) \cap$ $C\left(\mathbb{R}^{n}\right)$ (if $p=\infty$ ) for which the norm

$$
\|f\|_{B_{p, r}^{\sigma, \alpha}}=\|f\|_{p}+\left\|t^{-\sigma-1 / r} \ell^{\alpha}(t) \omega_{M}(f, t)_{p}\right\|_{r ;(0, \infty)}
$$

is finite. (Note that the classical Besov space $B_{p, r}^{\sigma}\left(\mathbb{R}^{n}\right)$ is obtained on putting $\alpha=0$.)

\subsection{Equivalent norms in Besov spaces}

It follows directly from (1) that an equivalent norm results on $B_{p, r}^{\sigma, \alpha}\left(\mathbb{R}^{n}\right)$ if the range $(0, \infty)$ of $t$ in $(2)$ is replaced by $(0,1)$. Equivalent norms result also from different choices of integers $M>\sigma$. This is a corollary of the Marchaud theorem:

Theorem 1.1 ([3, Thm. 4.4, Chap. 5]). If $k$ and $m$ are integers satisfying $0<k<m$, then, for all $t>0$,

$$
2^{k-m} \omega_{m}(f, t)_{p} \leq \omega_{k}(f, t)_{p} \lesssim t^{k} \int_{t}^{\infty} \frac{\omega_{m}(f, u)_{p}}{u^{k}} \frac{\mathrm{d} u}{u}
$$

\section{Main results}

Our main results are Theorems 2.1 and 2.3.

Theorem 2.1. Let $\kappa \in \mathfrak{M}^{+}(0,1 ; \downarrow), 1 \leq r \leq s \leq \infty, 1 \leq p<\infty, 0<\sigma<n / p$, $1 / q=1 / p-\sigma / n$ and $\alpha \in \mathbb{R}$. Then the following two statements are equivalent:

(i) There is a positive constant $C$ such that

$$
\left\|t^{1 / q-1 / s} \ell^{\alpha}(t) \kappa(t) f^{*}(t)\right\|_{s ;(0,1)} \leq C\|f\|_{B_{p, r}^{\sigma, \alpha}\left(\mathbb{R}^{n}\right)}
$$

for all $f \in B_{p, r}^{\sigma, \alpha}\left(\mathbb{R}^{n}\right)$.

(ii) The function $\kappa$ is bounded on $(0,1)$.

Remarks 2.2. (i) Putting $\alpha=0$ and $s=r$ in Theorem 2.1, we arrive at Theorem 2.3 of $[22]$. 
(ii) Let $\Omega \subset \mathbb{R}^{n}$ with $|\Omega|_{n}=1$. Taking $\kappa \equiv 1$ in Theorem 2.1, we obtain the embedding

$$
B_{p, r}^{\sigma, \alpha}\left(\mathbb{R}^{n}\right) \hookrightarrow L_{q, s ; \alpha}(\Omega) \quad \text { for all } s \in[r, \infty] .
$$

Moreover, Theorem 2.1 implies that embedding (4) is sharp. For example, if $Q \in(0, \infty), m \in \mathbb{N}, \alpha_{1}, \ldots, \alpha_{m} \in \mathbb{R}$ and

$$
B_{p, r}^{\sigma, \alpha}\left(\mathbb{R}^{n}\right) \hookrightarrow L_{Q, s ; \alpha_{1}, \alpha_{2}, \ldots, \alpha_{m}}(\Omega) \text { for some } s \in[r, \infty],
$$

then

$$
\begin{aligned}
\text { either } \quad q>Q ; \\
\text { or } \quad q=Q, \quad \alpha_{1}<\alpha ; \\
\text { or } \quad q=Q, \quad \alpha_{1}=\alpha, \quad \alpha_{2}<0 ; \\
\quad \vdots \\
\text { or } \quad q=Q, \quad \alpha_{1}=\alpha, \quad \alpha_{2}=0, \ldots, \quad \alpha_{m-2}=0, \quad \alpha_{m-1}<0 ; \\
\text { or } \quad q=Q, \quad \alpha_{1}=\alpha, \quad \alpha_{2}=0, \ldots, \quad \alpha_{m-2}=0, \quad \alpha_{m-1}=0, \quad \alpha_{m} \leq 0 .
\end{aligned}
$$

Since $L_{q, r ; \alpha_{1}, \alpha_{2}, \ldots, \alpha_{m}}(\Omega) \hookrightarrow L_{q, s ; \alpha_{1}, \alpha_{2}, \ldots, \alpha_{m}}(\Omega)$ if $s>r$, we see that the space $L_{q, r ; \alpha_{1}, \alpha_{2}, \ldots, \alpha_{m}}(\Omega)$ is the optimal (i.e. the smallest) target space for the embed$\operatorname{ding}(5)$.

(iii) Putting $\alpha=0, r=p$ and $s=q$ in (4), we obtain the embedding

$$
B_{p, p}^{\sigma}\left(\mathbb{R}^{n}\right) \hookrightarrow L^{q}(\Omega),
$$

which corresponds to the Sobolev embedding theorem.

Theorem 2.3. Let $\kappa \in \mathfrak{M}^{+}(0,1 ; \downarrow), 1 \leq r \leq s \leq \infty, 1<p<\infty$, and either $\delta<0$, or $\delta \leq 0$ when $r=1$ and $s=\infty$. Then the following two statements are equivalent:

(i) There is a positive constant $C$ such that

$$
\left\|t^{-1 / s} \ell^{\delta-1 / s}(t) \kappa(t) f^{*}(t)\right\|_{s ;(0,1)} \leq C\|f\|_{B_{p, r}^{n / p, \delta+1 / r^{\prime}}\left(\mathbb{R}^{n}\right)}
$$

for all $f \in B_{p, r}^{n / p, \delta+1 / r^{\prime}}\left(\mathbb{R}^{n}\right)$.

(ii) The function $\kappa$ is bounded on $(0,1)$.

Remarks 2.4. (i) Putting $p=r=s \in(1, \infty)$ and $\delta=-1 / r^{\prime}$ in Theorem 2.3, we arrive at Theorem 2.5 (with $A_{p}=B_{p, p}^{n / p}$ ) of [15].

(ii) Let $\Omega \subset \mathbb{R}^{n}$ with $|\Omega|_{n}=1$. Taking $\kappa \equiv 1$ in Theorem 2.3, we obtain the embedding

$$
B_{p, r}^{n / p, \delta+1 / r^{\prime}}\left(\mathbb{R}^{n}\right) \hookrightarrow L_{\infty, s ; \delta-1 / s}(\Omega) .
$$

Moreover, by Theorem 2.3, this embedding is sharp. 
(iii) Putting $s=r$ in (7), we obtain that

$$
B_{p, r}^{n / p, \delta+1 / r^{\prime}}\left(\mathbb{R}^{n}\right) \hookrightarrow L_{\infty, r ; \delta-1 / r}(\Omega)
$$

for any $\Omega \subset \mathbb{R}^{n}$ with $|\Omega|_{n}=1$. In addition, if $r>1$ and $\delta=-1 / r^{\prime}$, we arrive at the embedding

$$
B_{p, r}^{n / p}\left(\mathbb{R}^{n}\right) \hookrightarrow L_{\infty, r ;-1}(\Omega),
$$

which is an analogue of the result from [4].

(iv) Putting $s=\infty$ and $\kappa \equiv 1$ in (6), we obtain that

$$
B_{p, r}^{n / p, \delta+1 / r^{\prime}}\left(\mathbb{R}^{n}\right) \hookrightarrow L_{\infty, \infty ; \delta}(\Omega)
$$

for any $\Omega \subset \mathbb{R}^{n}$ with $|\Omega|_{n}=1$. In particular, if $r>1$ and $\delta=-1 / r^{\prime}$, we arrive at

$$
B_{p, r}^{n / p}\left(\mathbb{R}^{n}\right) \hookrightarrow L_{\infty, \infty ;-1 / r^{\prime}}(\Omega)=\operatorname{EXP} L^{r^{\prime}}(\Omega),
$$

which is the result of [21] (and an analogue of the embedding from [24]). Moreover, the choice $r=1$ and $\delta=-1 / r^{\prime}=0$ in (8) yields the well-known embedding

$$
B_{p, 1}^{n / p}\left(\mathbb{R}^{n}\right) \hookrightarrow L^{\infty}(\Omega) .
$$

Remarks 2.5. (i) If we use an analogue of the terminology from [23, section 12], then we can see from Theorem 2.1 that the function

$$
t \mapsto t^{-1 / q} \ell^{-\alpha}(t), \quad t \in(0, \varepsilon),
$$

where $\varepsilon \in(0,1)$ is a given number, dominates the growth envelope function $\mathcal{E}_{G} \mid B_{p, r}^{\sigma, \alpha}$ of the space $B_{p, r}^{\sigma, \alpha}\left(\mathbb{R}^{n}\right)$ if

$$
\begin{aligned}
& 1 \leq r \leq \infty, \quad 1 \leq p<\infty, \quad 0<\sigma<n / p, \quad \alpha \in \mathbb{R}, \\
& \text { and } 1 / q=1 / p-\sigma / n .
\end{aligned}
$$

Moreover, using the test function $h:=f /\|f\|_{B_{p, r}^{\sigma, \alpha}}$ with $f=f_{R}$ from Lemma 3.8 below, one can prove the reverse estimate (see the Appendix). Furthermore, in the Appendix we show that inequality (3) does not hold when $\kappa \equiv 1$ and $s \in(0, r)$. Consequently, the couple $\left(t^{-1 / q} \ell^{-\alpha}(t), r\right)$ is the growth envelope for the space $B_{p, r}^{\sigma, \alpha}\left(\mathbb{R}^{n}\right)$, provided that (10) is satisfied.

(ii) Similarly, we can see from Theorem 2.3 that the function

$$
t \mapsto \ell^{-\delta}(t), \quad t \in(0, \varepsilon),
$$

where $\varepsilon \in(0,1)$ is a given number, dominates the growth envelope function $\mathcal{E}_{G} \mid B_{p, r}^{n / p, \delta+1 / r^{\prime}}$ of the space $B_{p, r}^{n / p, \delta+1 / r^{\prime}}\left(\mathbb{R}^{n}\right)$ if

$$
1 \leq r \leq \infty, \quad 1<p<\infty, \quad \delta<0 .
$$


Making use of the test function $h:=f /\|f\|_{B_{p, r}^{n / p, \delta+1 / r^{\prime}}}$ with $f=f_{R}$ from Lemma 4.7 below, one can verify the reverse estimate (see the Appendix). Moreover, in the Appendix it is shown that the inequality (6) does not hold with any $s \in(0, r)$ when $\kappa \equiv 1$ and $\delta<0$. Consequently, the couple $\left(\ell^{-\delta}(t), r\right)$ is the growth envelope for the space $B_{p, r}^{n / p, \delta+1 / r^{\prime}}\left(\mathbb{R}^{n}\right)$ provided that (12) is satisfied.

(iii) In particular, if $\alpha=0$ and $\delta=-1 / r^{\prime}<0$, respectively, in part (i) and (ii) of this remark, then our spaces become the classical Besov spaces and our results on the growth envelopes coincide with those of [23]. On the other hand, our results on the growth envelopes of Besov spaces with logarithmic smoothness are particular cases of $[5,6]$, where the growth envelopes of Besov spaces of generalized smoothness are established. Note also that the approach of $[5,6]$ is completely different from that of ours; they use atomic decomposition to get their results.

\section{Proof of Theorem 2.1}

First, to prove the inequality (3), we shall make use of the following lemma which gives a relationship between the non-increasing rearrangement and the modulus of continuity.

Lemma 3.1 ([3, Thm. 4.19, chap. 5]). Let $1 \leq p<\infty$. Then, for all $f \in L^{p}\left(\mathbb{R}^{n}\right)$ and every $t>0$,

$$
f^{* *}(t) \lesssim \int_{t^{1 / n}}^{\infty} \frac{\omega_{n}(f, y)_{p}}{y^{n / p}} \frac{\mathrm{d} y}{y}
$$

Furthermore, we shall use a convenient version of the Hardy inequality.

Lemma 3.2 ([14, Lemma 4.1(ii)]). Let $1 \leq r \leq s \leq \infty, \beta, \delta, \nu \in \mathbb{R}$ and $\nu \neq 0$. Then the inequality

$$
\left\|t^{\nu-1 / s} \ell^{\delta}(t) \int_{t}^{\infty} g(u) \mathrm{d} u\right\|_{s ;(0, \infty)} \lesssim\left\|t^{\nu+1 / r^{\prime}} \ell^{\beta}(t) g(t)\right\|_{r ;(0, \infty)}
$$

holds for all $g \in \mathfrak{M}^{+}(0, \infty)$ if and only if $\nu>0$ and $\delta \leq \beta$.

Proof of the implication (ii) $\Rightarrow$ (i). Clearly, it is enough to prove (3) with $\kappa \equiv 1$. To this end, assume that $f \in B_{p, r}^{\sigma, \alpha}\left(\mathbb{R}^{n}\right)$. Applying (13), the change of variables $\tau=t^{1 / n}$, (14) (with $\nu=n / q, \delta=\beta=\alpha$ and $\left.g(t)=t^{-1-n / p} \omega_{n}(f, t)_{p}\right)$ and the equality 
$1 / q=1 / p-\sigma / n$, we obtain

$$
\begin{aligned}
\|f\|_{q, s ; \alpha} & =\left\|t^{1 / q-1 / s} \ell^{\alpha}(t) f^{*}(t)\right\|_{s ;(0, \infty)} \lesssim\left\|t^{1 / q-1 / s} \ell^{\alpha}(t) \int_{t^{1 / n}}^{\infty} \frac{\omega_{n}(f, y)_{p}}{y^{n / p}} \frac{\mathrm{d} y}{y}\right\|_{s ;(0, \infty)} \\
& \approx\left\|\tau^{n / q-1 / s} \ell^{\alpha}(\tau) \int_{\tau}^{\infty} \frac{\omega_{n}(f, y)_{p}}{y^{n / p}} \frac{\mathrm{d} y}{y}\right\|_{s ;(0, \infty)} \\
& \lesssim\left\|\tau^{n / q+1 / r^{\prime}} \ell^{\alpha}(\tau) \frac{\omega_{n}(f, \tau)_{p}}{\tau^{n / p+1}}\right\|_{r ;(0, \infty)}=\left\|\tau^{-\sigma-1 / r} \ell^{\alpha}(\tau) \omega_{n}(f, \tau)_{p}\right\|_{r ;(0, \infty)} \\
& \leq\|f\|_{B_{p, r}^{\sigma, \alpha}\left(\mathbb{R}^{n}\right)}
\end{aligned}
$$

The inequality (3) with $\kappa \equiv 1$ is a consequence of the previous estimate.

Now we turn our attention to the proof of the converse implication. We shall start with some auxiliary results.

Lemma 3.3. Let $1 \leq p \leq \infty$ and $M \in \mathbb{N}$. Let $F:[0, \infty) \rightarrow[0, \infty)$ and assume that the derivative of $F$ of order $(M-1)$ is absolutely continuous on any subinterval $[0, b] \subset[0, \infty)$. Put $f(x)=F(|x|), x \in \mathbb{R}^{n}$. Then there is a positive constant $c_{0}=c_{0}(M, n, p)$ such that, for all $h \in \mathbb{R}^{n} \backslash\{0\}$,

$$
\begin{aligned}
& \left\|\Delta_{h}^{M} f\right\|_{p ; \mathbb{R}^{n}} \\
\leq & c_{0}\left[\left\|F(\rho) \rho^{(n-1) / p}\right\|_{p ;(0,3 M|h|)}+|h|^{M} \sum_{k=1}^{M}\left\|F^{(k)}(\rho) \rho^{k-M+(n-1) / p}\right\|_{p ;(M|h|, \infty)}\right]
\end{aligned}
$$

(here $F^{(k)}$ stands for the derivative of $F$ of order $k$ ).

Remarks 3.4. Let all the assumptions of Lemma 3.3 be satisfied.

(i) Let $M<1+n / p$. Suppose that

$$
\lim _{\rho \rightarrow \infty} F^{(k)}(\rho)=0 \quad \text { for all } k \in\{1, \ldots, M-1\} \text { when } M>1 \text {. }
$$

Then there is a positive constant $c_{0}=c_{0}(M, n, p)$ such that, for all $h \in \mathbb{R}^{n} \backslash\{0\}$,

$$
\begin{aligned}
& \left\|\Delta_{h}^{M} f\right\|_{p ; \mathbb{R}^{n}} \\
& \quad \leq c_{0}\left[\left\|F(\rho) \rho^{(n-1) / p}\right\|_{p ;(0,3 M|h|)}+|h|^{M}\left\|F^{(M)}(\rho) \rho^{(n-1) / p}\right\|_{p ;(M|h|, \infty)}\right] .
\end{aligned}
$$

Indeed, the result follows from Lemma 3.3 when $M=1$. Thus, assume that $1<M<1+n / p$. Let $t \in(0, \infty)$ and $k \in\{1, \ldots, M-1\}$. Since the derivative $F^{(k)}$ is absolutely continuous on any subinterval $[0, b] \subset[0, \infty),(16)$ implies that

$$
F^{(k)}(\rho)=-\int_{\rho}^{\infty} F^{(k+1)}(\tau) \mathrm{d} \tau \quad \text { for all } \rho \in[0, \infty) .
$$


Hence,

$$
\left\|F^{(k)}(\rho) \rho^{k-M+(n-1) / p}\right\|_{p ;(t, \infty)}=\left\|\left(\int_{\rho}^{\infty} F^{(k+1)}\right) \rho^{k-M+(n-1) / p}\right\|_{p ;(t, \infty)} .
$$

The assumption $M<1+n / p$ guarantees that $k-M+n / p>0$. Thus, there is a positive constant $c=c(k, M, n, p)$ such that the Hardy inequality

$$
\begin{aligned}
\left\|\left(\int_{\rho}^{\infty} F^{(k+1)}\right) \rho^{k-M+(n-1) / p}\right\|_{p ;(t, \infty)} & \\
& \leq c\left\|F^{(k+1)}(\rho) \rho^{k+1-M+(n-1) / p}\right\|_{p ;(t, \infty)}
\end{aligned}
$$

holds (cf. [18]). Consequently, putting $C=\max _{j \in\{1, \ldots, M-1\}} c(j, M, n, p)$, we arrive at

$$
\sum_{k=1}^{M-1}\left\|F^{(k)}(\rho) \rho^{k-M+(n-1) / p}\right\|_{p ;(t, \infty)} \leq\left(\sum_{k=1}^{M-1} C^{M-k}\right)\left\|F^{(M)}(\rho) \rho^{(n-1) / p}\right\|_{p ;(t, \infty)}
$$

which means that (17) follows from (15).

(ii) Let $M \leq 1+n / p$ and let (16) hold. Then there is a positive constant $c_{0}=$ $c_{0}(M, n, p)$ such that, for all $h \in \mathbb{R}^{n} \backslash\{0\}$,

$$
\begin{aligned}
\left\|\Delta_{h}^{M} f\right\|_{p ; \mathbb{R}^{n}} \leq c_{0}[ & \left\|F(\rho) \rho^{(n-1) / p}\right\|_{p ;(0,3 M|h|)} \\
& +|h|^{M}\left(\left\|F^{\prime}(\rho) \rho^{1-M+(n-1) / p}\right\|_{p ;(M|h|, \infty)}\right. \\
& \left.\left.+\left\|F^{(M)}(\rho) \rho^{(n-1) / p}\right\|_{p ;(M|h|, \infty)}\right)\right] .
\end{aligned}
$$

Indeed, if $M \in\{1,2\}$, then (20) coincides with (15) and Lemma 3.3 yields the result. Thus, suppose that $2<M \leq 1+n / p$. The assumption $M \leq 1+n / p$ guarantees that $k-M+n / p>0$ for all $k \in\{2, \ldots, M-1\}$. Consequently, (20) follows on using (18) and Hardy inequalities (19) (with $t=M|h|$ ) for all $k \in\{2, \ldots, M-1\}$ to estimate $\sum_{k=2}^{M-1}\left\|F^{(k)}(\rho) \rho^{k-M+(n-1) / p}\right\|_{p ;(M|h|, \infty)}$ on the right-hand side of (15).

(iii) In particular, (16) holds if $F$ has a compact support.

Proof of Lemma 3.3. Assume that $1 \leq p<\infty$ (when $p=\infty$, the proof is similar and is left to the reader). Since (cf., for example, [3, p. 332])

$$
\Delta_{h}^{M} f(x)=\sum_{k=0}^{M}\left(\begin{array}{c}
M \\
k
\end{array}\right)(-1)^{M-k} f(x+k h), \quad x \in \mathbb{R}^{n},
$$


we have

$$
\begin{aligned}
\int_{|x| \leq 2 M|h|}\left|\Delta_{h}^{M} f(x)\right|^{p} \mathrm{~d} x & \leq(M+1)^{p-1} \sum_{k=0}^{M}\left(\begin{array}{c}
M \\
k
\end{array}\right)^{p} \int_{|x| \leq 2 M|h|}|f(x+k h)|^{p} \mathrm{~d} x \\
& \leq(M+1)^{p-1} \sum_{k=0}^{M}\left(\begin{array}{c}
M \\
k
\end{array}\right)^{p} \int_{|y| \leq 3 M|h|}|f(y)|^{p} \mathrm{~d} y .
\end{aligned}
$$

Hence, using spherical coordinates, we arrive at

$$
\int_{|x| \leq 2 M|h|}\left|\Delta_{h}^{M} f(x)\right|^{p} \mathrm{~d} x \leq c_{1} \int_{0}^{3 M|h|}|F(\rho)|^{p} \rho^{n-1} \mathrm{~d} \rho,
$$

where $c_{1}=c_{1}(M, n, p)$ is a positive constant.

Furthermore, one can easily show that, for all $x \in \mathbb{R}^{n}$,

$$
\left|\Delta_{h}^{M} f(x)\right| \leq|h|^{M} \sum_{|\gamma|=M} \int_{\mathcal{I}_{M}}\left|D^{\gamma} f\left(x+\left(\tau_{1}+\cdots+\tau_{M}\right) h\right)\right| \mathrm{d} \tau_{1} \cdots \mathrm{d} \tau_{M},
$$

where $\gamma=\left(\gamma_{1}, \ldots, \gamma_{n}\right)$ is a multiindex, $|\gamma|:=\gamma_{1}+\cdots+\gamma_{n}$ is its length, and $\mathcal{I}_{M}=(0,1)^{M}$.

Applying Hölder's inequality (if $p>1$ ) and then Fubini's theorem, we obtain from (23) that

$$
\begin{aligned}
& \int_{|x|>2 M|h|}\left|\Delta_{h}^{M} f(x)\right|^{p} \mathrm{~d} x \\
& \leq c_{2}|h|^{M p} \sum_{|\gamma|=M} \int_{\mathcal{I}_{M}}\left(\int_{|x|>2 M|h|}\left|D^{\gamma} f\left(x+\left(\tau_{1}+\cdots+\tau_{M}\right) h\right)\right|^{p} \mathrm{~d} x\right) \mathrm{d} \tau_{1} \cdots \mathrm{d} \tau_{M} \\
& \leq c_{2}|h|^{M p} \sum_{|\gamma|=M} \int_{\mathcal{I}_{M}}\left(\int_{|y|>M|h|}\left|D^{\gamma} f(y)\right|^{p} \mathrm{~d} y\right) \mathrm{d} \tau_{1} \cdots \mathrm{d} \tau_{M},
\end{aligned}
$$

where $c_{2}=c_{2}(M, n, p)$ is a positive constant. Thus, using spherical coordinates and the estimate

$$
\left|D^{\gamma} f(y)\right| \leq c_{3} \sum_{k=1}^{M}\left|F^{(k)}(\rho)\right| \rho^{k-M} \quad \text { if } \quad|y|=\rho \text { and }|\gamma|=M,
$$

we arrive at

$$
\int_{|x|>2 M|h|}\left|\Delta_{h}^{M} f(x)\right|^{p} \mathrm{~d} x \leq c_{4}|h|^{M p} \sum_{k=1}^{M} \int_{M|h|}^{\infty}\left|F^{(k)}(\rho)\right|^{p} \rho^{(k-M) p+n-1} \mathrm{~d} \rho,
$$


where $c_{3}=c_{3}(M, n, p)$ and $c_{4}=c_{4}(M, n, p)$ are positive constants.

The inequality (15) is a consequence of estimates (22) and (24).

Now we make an appropriate choice of the function $F$ appearing in the previous lemma. We assume that

$$
\begin{gathered}
1 \leq p<\infty, \quad 0<\sigma<n / p, \quad M \in \mathbb{N}, \\
M \in[n / p, 1+n / p), \quad \text { and } \quad \gamma \in(-n / p, \sigma-n / p) .
\end{gathered}
$$

Taking a fixed $R \in(0,1)$, we define the functions

$$
g(t)=g_{R}(t)=t^{\gamma-M} \chi_{(R, 1)}(t), \quad t \in \mathbb{R},
$$

and

$$
F(\rho)=F_{R}(\rho)=\frac{1}{(M-1) !} \int_{\rho}^{\infty}(t-\rho)^{M-1} g(t) \mathrm{d} t, \quad \rho \in[0, \infty) .
$$

Obviously,

$$
\left|F^{(M)}(\rho)\right|=g(\rho), \quad \rho \in(0, \infty) .
$$

Moreover, we have the following assertion.

Lemma 3.5. Suppose that (25) holds. Let $F$ be given by (26), (27). Then there is a positive constant $c=c(\gamma, M, n, p)$ such that

$$
F(\rho) \leq\left\{\begin{array}{lll}
0 & \text { if } & \rho \in[1, \infty) \\
c \rho^{\gamma} & \text { if } & \rho \in[R, 1) \\
c R^{\gamma} & \text { if } & \rho \in[0, R)
\end{array}\right.
$$

and

$$
\begin{aligned}
\left\|F(\rho) \rho^{(n-1) / p}\right\|_{p ;(0, t)} & \leq c R^{\gamma} t^{n / p} \\
\left\|F^{(M)}(\rho) \rho^{(n-1) / p}\right\|_{p ;(t, \infty)} & \leq c R^{\gamma-M+n / p}
\end{aligned}
$$

for all $t \in(0, R]$, and

$$
\begin{aligned}
\left\|F(\rho) \rho^{(n-1) / p}\right\|_{p ;(0, t)} & \leq c[\min \{t, 1\}]^{\gamma+n / p}, \\
\left\|F^{(M)}(\rho) \rho^{(n-1) / p}\right\|_{p ;(t, \infty)} & \leq c t^{\gamma-M+n / p} \chi_{(R, 1)}(t)
\end{aligned}
$$

for all $t \in(R, \infty)$.

Proof. Since

$$
F(\rho) \leq \frac{1}{(M-1) !} \int_{(\rho, \infty) \cap(R, 1)} t^{\gamma-1} \mathrm{~d} t, \quad \rho \in[0, \infty)
$$


and $\gamma<0$ by (25), the estimate (29) follows (with $c=c(\gamma, M)$ ).

Now, let $t \in(0, R]$. Then, by (29),

$$
\left\|F(\rho) \rho^{(n-1) / p}\right\|_{p ;(0, t)} \leq c R^{\gamma}\left\|\rho^{(n-1) / p}\right\|_{p ;(0, t)} \leq c n^{-1 / p} R^{\gamma} t^{n / p},
$$

which gives (30). Moreover, (28), the equality $\operatorname{supp} g=[R, 1]$, and (26) imply that

$$
\begin{aligned}
\left\|F^{(M)}(\rho) \rho^{(n-1) / p}\right\|_{p ;(t, \infty)} & \leq\left\|g(\rho) \rho^{(n-1) / p}\right\|_{p ;(t, R)}+\left\|g(\rho) \rho^{(n-1) / p}\right\|_{p ;(R, \infty)} \\
& =\left\|\rho^{\gamma-M} \rho^{(n-1) / p}\right\|_{p ;(R, 1)} .
\end{aligned}
$$

According to (25), $\gamma-M+n / p<0$. Consequently,

$$
\left\|\rho^{(\gamma-M+n / p)-1 / p}\right\|_{p ;(R, 1)} \leq[(M-\gamma) p-n]^{-1 / p} R^{\gamma-M+n / p}
$$

and (31) follows from (34) and (35).

Suppose that $t \in(R, 1)$. Then

$$
\left\|F(\rho) \rho^{(n-1) / p}\right\|_{p ;(0, t)} \leq\left\|F(\rho) \rho^{(n-1) / p}\right\|_{p ;(0, R)}+\left\|F(\rho) \rho^{(n-1) / p}\right\|_{p ;(R, t)} .
$$

By (30),

$$
\left\|F(\rho) \rho^{(n-1) / p}\right\|_{p ;(0, R)} \leq c R^{\gamma+n / p} .
$$

On the other hand, using (29) and the inequality $\gamma+n / p>0$ (cf. (25)), we see that

$$
\begin{aligned}
\left\|F(\rho) \rho^{(n-1) / p}\right\|_{p ;(R, t)} & \leq c\left\|\rho^{\gamma} \rho^{(n-1) / p}\right\|_{p ;(R, t)} \\
& \leq c\left\|\rho^{(\gamma+n / p)-1 / p}\right\|_{p ;(0, t)}=c[\gamma p+n]^{-1 / p} t^{\gamma+n / p} .
\end{aligned}
$$

The estimate (32) is a consequence of (36)-(38). Moreover, (28), the fact that $\operatorname{supp} g=[R, 1]$, and $(26)$ yield

$$
\begin{aligned}
\left\|F^{(M)}(\rho) \rho^{(n-1) / p}\right\|_{p ;(t, \infty)} & =\left\|g(\rho) \rho^{(n-1) / p}\right\|_{p ;(t, 1)} \\
& =\left\|\rho^{\gamma-M} \rho^{(n-1) / p}\right\|_{p ;(t, 1)} \leq[(M-\gamma) p-n]^{-1 / p} t^{\gamma-M+n / p}
\end{aligned}
$$

(cf. (35)), and (33) is verified.

Finally, let $t \in[1, \infty)$. Then, since $\operatorname{supp} F=[0,1]$,

$$
\left\|F(\rho) \rho^{(n-1) / p}\right\|_{p ;(0, t)}=\left\|F(\rho) \rho^{(n-1) / p}\right\|_{p ;(0,1)} \leq c[\gamma p+n]^{-1 / p}
$$

(cf. (36)-(38)), and

$$
\left\|F^{(M)}(\rho) \rho^{(n-1) / p}\right\|_{p ;(t, \infty)}=0 .
$$

Thus, (32) and (33) again hold.

Lemma 3.6. Suppose that (25) holds. Let $f(x)=F(|x|)=F_{R}(|x|), x \in \mathbb{R}^{n}$, where the function $F$ is given by $(26)-(27)$. Then there is a positive constant $C=$ $C(\gamma, M, n, p)$ such that, for all $\tau \in(0,1)$,

$$
\omega_{M}(f, \tau)_{p} \leq C\left[R^{\gamma} \tau^{n / p} \chi_{(0, R]}(\tau)+\tau^{\gamma+n / p} \chi_{(R, 1)}(\tau)\right] .
$$


Proof. Let $h \in \mathbb{R}^{n}, 0<|h|<1$. Then, by Remarks 3.4 (i) and (iii), the inequality (17) is satisfied, that is,

$$
\left\|\Delta_{h}^{M} f\right\|_{p ; \mathbb{R}^{n}} \leq c_{0}\left[N_{1}(|h|)+N_{2}(|h|)\right]
$$

where

$$
\begin{aligned}
& N_{1}(\tau):=\left\|F(\rho) \rho^{(n-1) / p}\right\|_{p ;(0,3 M \tau)}, \\
& N_{2}(\tau):=\tau^{M}\left\|F^{(M)}(\rho) \rho^{(n-1) / p}\right\|_{p ;(M \tau, \infty)}
\end{aligned}
$$

for $\tau \in(0,1)$. By $(30)-(33)$,

$$
N_{1}(\tau) \leq\left\{\begin{array}{lll}
c_{1} R^{\gamma} \tau^{n / p} & \text { if } & 0<\tau \leq R /(3 M), \\
c_{1} \tau^{\gamma+n / p} & \text { if } & R /(3 M)<\tau<1
\end{array}\right.
$$

and

$$
N_{2}(\tau) \leq\left\{\begin{array}{lll}
c_{1} R^{\gamma-M+n / p} \tau^{M} & \text { if } & 0 \leq \tau \leq R / M \\
c_{1} \tau^{\gamma+n / p} & \text { if } & R / M<\tau<1,
\end{array}\right.
$$

where $c_{1}=c_{1}(\gamma, M, n, p)$ is a suitable positive constant. These estimates immediately imply that

$$
N_{1}(\tau)+N_{2}(\tau) \leq 2 c_{1} \tau^{\gamma+n / p} \quad \text { if } \quad R<\tau<1 .
$$

Since $M-n / p \geq 0$ (cf. (25)), we have $\tau^{M}=\tau^{M-n / p} \tau^{n / p} \leq R^{M-n / p} \tau^{n / p}$ for all $\tau \in(0, R]$, which, together with (42), implies that

$$
N_{2}(\tau) \leq c_{1} R^{\gamma} \tau^{n / p} \quad \text { if } \quad 0<\tau \leq R / M
$$

Moreover, since $\gamma<0$ (cf. (25)), we have $R^{\gamma} \leq \tau^{\gamma}<(3 M)^{-\gamma} R^{\gamma}$ for all $\tau \in$ $(R /(3 M), R]$. Consequently,

$$
N_{2}(\tau) \leq c_{1} R^{\gamma} \tau^{n / p} \quad \text { if } \quad R / M<\tau \leq R,
$$

and

$$
N_{1}(\tau) \leq c_{1}(3 M)^{-\gamma} R^{\gamma} \tau^{n / p} \quad \text { if } \quad R /(3 M)<\tau \leq R .
$$

The last estimates, (44), and (41) imply that

$$
N_{1}(\tau)+N_{2}(\tau) \leq c_{2} R^{\gamma} \tau^{n / p} \quad \text { if } \quad 0<\tau \leq R,
$$

where $c_{2}=c_{1}\left[(3 M)^{-\gamma}+1\right]$. Together with (43), this yields

$$
N_{1}(\tau)+N_{2}(\tau) \leq c_{2} N(\tau), \quad \tau \in(0,1),
$$

where

$$
N(\tau):=R^{\gamma} \tau^{n / p} \chi_{(0, R]}(\tau)+\tau^{\gamma+n / p} \chi_{(R, 1)}(\tau) .
$$

This estimate, the fact that the function $N$ is increasing on the interval $(0,1)$, and (40) imply (39). 
Now, we are able to estimate (from above) the norm of the test function $f=f_{R}$ in the space $B_{p, r}^{\sigma, \alpha}\left(\mathbb{R}^{n}\right)$.

Lemma 3.7. Suppose that (25) holds, $1 \leq r \leq \infty$ and $\alpha \in \mathbb{R}$. Let $f(x)=F_{R}(|x|)$, $x \in \mathbb{R}^{n}$, where the function $F_{R}$ is given by (26), (27). Then there is a positive constant $\bar{c}$ independent of $R$ such that, for all $R \in(0,1)$,

$$
\|f\|_{B_{p, r}^{\sigma, \alpha}} \leq \bar{c} R^{\gamma-\sigma+n / p} \ell^{\alpha}(R) .
$$

Proof. Using spherical coordinates, the estimate (29), the conditions $\gamma+n / p>0$ and $\gamma-\sigma+n / p<0($ cf. $(25))$, we obtain

$$
\begin{aligned}
\|f\|_{p} & =s_{n}^{1 / p}\left\|F(\rho) \rho^{(n-1) / p}\right\|_{p ;(0, \infty)} \\
& \leq s_{n}^{1 / p}\left[\left\|F(\rho) \rho^{(n-1) / p}\right\|_{p ;(0, R)}+\left\|F(\rho) \rho^{(n-1) / p}\right\|_{p ;(R, \infty)}\right] \\
& \leq s_{n}^{1 / p} c\left[R^{\gamma}\left\|\rho^{(n-1) / p}\right\|_{p ;(0, R)}+\left\|\rho^{\gamma+(n-1) / p}\right\|_{p ;(R, 1)}\right] \\
& \leq s_{n}^{1 / p} c\left[n^{-1 / p} R^{\gamma+n / p}+\left\|\rho^{\gamma+(n-1) / p}\right\|_{p ;(0,1)}\right] \\
& \leq s_{n}^{1 / p} c\left[n^{-1 / p}+(\gamma p+n)^{-1 / p}\right] \leq \bar{c} R^{\gamma-\sigma+n / p} \ell^{\alpha}(R)
\end{aligned}
$$

for all $R \in(0,1)$, where $\bar{c}$ is a positive constant independent of $R$.

Furthermore, putting

$$
\mathcal{I}_{1}=(0, R] \quad \text { and } \quad \mathcal{I}_{2}=(R, 1),
$$

we have

$$
\left\|t^{-\sigma-1 / r} \ell^{\alpha}(t) \omega_{M}(f, t)_{p}\right\|_{r ;(0,1)} \leq N_{1}+N_{2},
$$

where

$$
N_{i}:=\left\|t^{-\sigma-1 / r} \ell^{\alpha}(t) \omega_{M}(f, t)_{p}\right\|_{r ; \mathcal{I}_{i}}, \quad i=1,2 .
$$

Applying (39), we arrive at

$$
N_{1} \leq C R^{\gamma}\left\|t^{-\sigma-1 / r} \ell^{\alpha}(t) t^{n / p}\right\|_{r ; \mathcal{I}_{1}} \approx R^{\gamma-\sigma+n / p} \ell^{\alpha}(R)
$$

for all $R \in(0,1)$. Similarly, we obtain

$$
N_{2} \leq C\left\|t^{-\sigma-1 / r} \ell^{\alpha}(t) t^{\gamma+n / p}\right\|_{r ; \mathcal{I}_{2}} \lesssim R^{\gamma-\sigma+n / p} \ell^{\alpha}(R)
$$

for all $R \in(0,1)$. The estimate (45) is a consequence of (46)-(49).

The next lemma gives an estimate from below of the left-hand side of inequality (3) with an appropriate choice of a function $f$.

Lemma 3.8. Suppose that (25) holds. Let $f(x)=F(|x|)=F_{R}(|x|), x \in \mathbb{R}^{n}$, where the function $F_{R}$ is given by (26)-(27). Let $\kappa \in \mathfrak{M}^{+}(0,1 ; \downarrow), 1 / q=1 / p-\sigma / n$, $1 \leq s \leq \infty$ and $\alpha \in \mathbb{R}$. Put $R_{0}=\min \left\{2^{-1+1 /(\gamma-M+1)}, \beta_{n}^{-1 / n}\right\}$. Then there is a positive constant $\bar{C}$ independent of $R$ such that, for all $R \in\left(0, R_{0}\right)$,

$$
\left\|t^{1 / q-1 / s} \ell^{\alpha}(t) \kappa(t) f^{*}(t)\right\|_{s ;(0,1)} \geq \bar{C} R^{\gamma-\sigma+n / p} \ell^{\alpha}(R) \kappa\left(\beta_{n} R^{n}\right) .
$$


Proof. Put $R_{1}=2^{-1+1 /(\gamma-M+1)}$. First we prove that there is a positive constant $c=c(\gamma, M)$ such that

$$
f(x) \geq c R^{\gamma} \chi_{(0, R)}(|x|) \text { for all } x \in \mathbb{R}^{n} \text { and } R \in\left(0, R_{1}\right) .
$$

Let $x \in \mathbb{R}^{n},|x|<R$ and $R \in\left(0, R_{1}\right)$. Then $2 R<1$ which, together with $(26)$ and (27), implies that

$$
f(x) \geq \frac{1}{(M-1) !} \int_{2 R}^{1}(t-|x|)^{M-1} t^{\gamma-M} \mathrm{~d} t \geq \frac{R^{M-1}}{(M-1) !} \int_{2 R}^{1} t^{\gamma-M} \mathrm{~d} t .
$$

Moreover, since $\gamma-M+1 \leq \gamma<0$ (cf. (25)), we have for all $R \in\left(0, R_{1}\right)$ that

$$
\int_{2 R}^{1} t^{\gamma-M} \mathrm{~d} t=(M-\gamma+1)^{-1}\left[(2 R)^{\gamma-M+1}-1\right] \geq[2(M-\gamma+1)]^{-1}(2 R)^{\gamma-M+1},
$$

and the estimate (51) follows.

The inequality (51) implies that

$$
f^{*}(t) \geq c R^{\gamma} \chi_{\left(0, \beta_{n} R^{n}\right)}(t) \quad \text { for all } t \in(0, \infty) \text { and } R \in\left(0, R_{1}\right)
$$

Consequently, for all $R \in\left(0, R_{0}\right)$,

$$
\begin{aligned}
\left\|t^{1 / q-1 / s} \ell^{\alpha}(t) \kappa(t) f^{*}(t)\right\|_{s ;(0,1)} & \geq c R^{\gamma}\left\|t^{1 / q-1 / s} \ell^{\alpha}(t) \kappa(t)\right\|_{s ;\left(0, \beta_{n} R^{n}\right)} \\
& \geq c R^{\gamma} \kappa\left(\beta_{n} R^{n}\right)\left\|t^{1 / q-1 / s} \ell^{\alpha}(t)\right\|_{s ;\left(0, \beta_{n} R^{n}\right)} \\
& \approx R^{\gamma} \kappa\left(\beta_{n} R^{n}\right) R^{n / q} \ell^{\alpha}(R) \\
& =R^{\gamma-\sigma+n / p} \ell^{\alpha}(R) \kappa\left(\beta_{n} R^{n}\right),
\end{aligned}
$$

and (50) is verified.

Now, we are able to prove the implication (i) $\Rightarrow$ (ii) in Theorem 2.1.

Proof of the implication (i) $\Rightarrow$ (ii). Let $f=f_{R}, R \in\left(0, R_{0}\right)$, be the function from Lemma 3.8. Then inequality (3) and estimates (45) and (50) imply that, for all $R \in\left(0, R_{0}\right)$,

$$
\bar{C} R^{\gamma-\sigma+n / p} \ell^{\alpha}(R) \kappa\left(\beta_{n} R^{n}\right) \leq C \bar{c} R^{\gamma-\sigma+n / p} \ell^{\alpha}(R)
$$

Consequently,

$$
\kappa\left(\beta_{n} R^{n}\right) \leq C \bar{c} / \bar{C} \quad \text { for all } R \in\left(0, R_{0}\right)
$$

and (ii) follows. 


\section{Proof of Theorem 2.3}

To prove (6) with $\kappa$ bounded on $(0,1)$, we shall need the following lemma.

Lemma 4.1 ([13, Lemma 3.2 (ii) and Rem. 3.4 (iii) $]$ ). Let $1 \leq r \leq s \leq \infty$ and $\delta \in \mathbb{R}$. Then the inequality

$$
\left\|t^{-1 / s} \ell^{\delta-1 / s}(t) \int_{t}^{1} g(u) \mathrm{d} u\right\|_{s ;(0,1)} \lesssim\left\|t^{1 / r^{\prime}} \ell^{\delta+1 / r^{\prime}}(t) g(t)\right\|_{r ;(0,1)}
$$

holds for all $g \in \mathfrak{M}^{+}(0,1)$ if and only if either $\delta<0$, or $\delta \leq 0$ when $r=1$ and $s=\infty$.

Proof of the implication (ii) $\Rightarrow$ (i). As in the previous section, it is enough to prove (6) with $\kappa \equiv 1$. Let $f \in B_{p, r}^{n / p, \delta+1 / r^{\prime}}\left(\mathbb{R}^{n}\right)$. Applying the inequality $f^{*} \leq f^{* *},(13)$, and the change of variables $\tau=t^{1 / n}$, we obtain

$$
\begin{aligned}
\left\|t^{-1 / s} \ell^{\delta-1 / s}(t) f^{*}(t)\right\|_{s ;(0,1)} \lesssim & \left\|t^{-1 / s} \ell^{\delta-1 / s}(t) \int_{t^{1 / n}}^{\infty} \frac{\omega_{n}(f, y)_{p}}{y^{n / p}} \frac{\mathrm{d} y}{y}\right\|_{s ;(0,1)} \\
\approx & \left\|\tau^{-1 / s} \ell^{\delta-1 / s}(\tau) \int_{\tau}^{\infty} \frac{\omega_{n}(f, y)_{p}}{y^{n / p}} \frac{\mathrm{d} y}{y}\right\|_{s ;(0,1)} \\
\lesssim & \left\|\tau^{-1 / s} \ell^{\delta-1 / s}(\tau) \int_{\tau}^{1} \frac{\omega_{n}(f, y)_{p}}{y^{n / p}} \frac{\mathrm{d} y}{y}\right\|_{s ;(0,1)} \\
& +\left\|\tau^{-1 / s} \ell^{\delta-1 / s}(\tau) \int_{1}^{\infty} \frac{\omega_{n}(f, y)_{p}}{y^{n / p}} \frac{\mathrm{d} y}{y}\right\|_{s ;(0,1)} \\
= & : N_{1}+N_{2} .
\end{aligned}
$$

Using Lemma $4.1\left(\right.$ with $\left.g(t)=t^{-1-n / p} \omega_{n}(f, t)_{p}\right)$, we arrive at

$$
\begin{aligned}
N_{1} & \lesssim\left\|\tau^{1 / r^{\prime}} \ell^{\delta+1 / r^{\prime}}(\tau) \frac{\omega_{n}(f, \tau)_{p}}{\tau^{n / p+1}}\right\|_{r ;(0,1)} \\
& =\left\|\tau^{-n / p-1 / r} \ell^{\delta+1 / r^{\prime}}(\tau) \omega_{n}(f, \tau)_{p}\right\|_{r ;(0,1)} \leq\|f\|_{B_{p, r}^{n / p, \delta+1 / r^{\prime}}}
\end{aligned}
$$

Moreover, by (1),

$$
\begin{aligned}
N_{2} & \lesssim\|f\|_{p}\left\|\tau^{-1 / s} \ell^{\delta-1 / s}(\tau) \int_{1}^{\infty} \frac{\mathrm{d} y}{y^{1+n / p}}\right\|_{s ;(0,1)} \\
& \approx\|f\|_{p}\left\|\tau^{-1 / s} \ell^{\delta-1 / s}(\tau)\right\|_{s ;(0,1)} \approx\|f\|_{p} \lesssim\|f\|_{B_{p, r}^{n, p}, \delta+1 / r^{\prime}}
\end{aligned}
$$

and (6) follows. 
Now we turn our attention to the proof of the converse implication. To this end, we need an appropriate test function $f$. Let us assume that

$$
\begin{gathered}
1<p<\infty, \quad M \in \mathbb{N}, \quad M \in(n / p, 1+n / p], \quad \theta<\delta \leq 0, \\
\text { and } \alpha \in(1, \infty) \text { is such that } 1 / \alpha^{\prime}<\min \{n / p, M-n / p\} .
\end{gathered}
$$

Take a fixed $R \in(0,1 / 2)$ and define $f$ by

$$
f(x)=f_{R}(x)=F(|x|), \quad x \in \mathbb{R}^{n},
$$

where the function $F=F_{R}$ is given on $[0, \infty)$ by

$$
F(\rho):= \begin{cases}\int_{\rho}^{\infty} \tau^{-1} \ell(\tau)^{-1-\theta} \chi_{(R, 1)}(\tau) \mathrm{d} \tau & \text { if } \quad M=1 \\ \int_{\rho}^{\infty} \psi_{R}(t) \int_{t}^{\infty}(\tau-t)^{M-2} \tau^{-M} \ell(\tau)^{-1-\theta} \chi_{(R, 1)}(\tau) \mathrm{d} \tau \mathrm{d} t & \text { if } \quad M \geq 2\end{cases}
$$

and $\psi_{R}$ is a $C^{\infty}(\mathbb{R})$ cut-off function such that $0 \leq \psi_{R} \leq 1$,

$$
\begin{aligned}
& \psi_{R}(t)=0 \quad \text { for } \quad t \leq R, \quad \psi_{R}(t)=1 \quad \text { for } \quad t \geq 2 R, \\
& \left|\frac{\mathrm{d}^{k}}{\mathrm{~d} t^{k}} \psi_{R}\right| \leq \tilde{c} R^{-k} \chi_{(R, 2 R)} \quad \text { if } \quad k \in \mathbb{N},
\end{aligned}
$$

with the constant $\tilde{c}$ independent of $R$. (See Remark 4.8 below for an explanation why the cut-off function $\psi_{R}$ is used in (55).)

In the whole paragraph, by $A \lesssim B$ we mean that $A \leq c B$, where $c$ is a positive constant which may depend only on the parameters $\alpha, \theta, M, n$, and $p$. Similarly for $A \gtrsim B$ and $A \approx B$.

We would like to have a suitable analogue of Lemma 3.6. Since Lemma 3.3 does not provide a sufficiently sharp estimate of $\left\|\Delta_{h}^{M} f\right\|_{p ; B}, B=\left\{x \in \mathbb{R}^{n}:|x| \leq 2 M|h|\right\}$, with $f$ given by (54) and (55), we shall look for a more precise one.

Lemma 4.2. Let $1 \leq p \leq \infty, M \in \mathbb{N}$ and $\alpha \in[1, \infty)$. Let $F:[0, \infty) \rightarrow[0, \infty)$ and assume that the derivative of $F$ of order $(M-1)$ is absolutely continuous on any subinterval $[0, b] \subset[0, \infty)$. Put $f(x)=F(|x|), x \in \mathbb{R}^{n}$. Then there is a positive constant $c_{0}=c_{0}(M, n, p)$ such that, for all $h \in \mathbb{R}^{n} \backslash\{0\}$,

$$
\begin{aligned}
\left\|\Delta_{h}^{M} f\right\|_{p ; \mathbb{R}^{n}} \leq & c_{0}\left[|h|^{1 / \alpha^{\prime}}\left\|\left(\int_{\rho}^{\infty}\left|F^{\prime}\right|^{\alpha}\right)^{1 / \alpha} \rho^{(n-1) / p}\right\|_{p ;(0,3 M|h|)}\right. \\
& \left.+|h|^{M} \sum_{k=1}^{M}\left\|F^{(k)}(\rho) \rho^{k-M+(n-1) / p}\right\|_{p ;(M|h|, \infty)}\right]
\end{aligned}
$$

(here $F^{\prime}$ is the first derivative of $F$ and $F^{(k)}$ stands for the $k$-th derivative of $F$ ).

The following remarks can be verified analogously as Remarks 3.4. 
Remarks 4.3. Let the assumptions of Lemma 4.2 be satisfied.

(i) Let $M<1+n / p$ and let (16) hold. Then there is a positive constant $c_{0}=c_{0}(M, n, p)$ such that, for all $h \in \mathbb{R}^{n} \backslash\{0\}$,

$$
\begin{aligned}
\left\|\Delta_{h}^{M} f\right\|_{p ; \mathbb{R}^{n}} \leq & c_{0}\left[|h|^{1 / \alpha^{\prime}}\left\|\left(\int_{\rho}^{\infty}\left|F^{\prime}\right|^{\alpha}\right)^{1 / \alpha} \rho^{(n-1) / p}\right\|_{p ;(0,3 M|h|)}\right. \\
& \left.+|h|^{M}\left\|F^{(M)}(\rho) \rho^{(n-1) / p}\right\|_{p ;(M|h|, \infty)}\right] .
\end{aligned}
$$

(ii) Let $M \leq 1+n / p$ and let (16) hold. Then there is a positive constant $c_{0}=$ $c_{0}(M, n, p)$ such that, for all $h \in \mathbb{R}^{n} \backslash\{0\}$,

$$
\begin{aligned}
\left\|\Delta_{h}^{M} f\right\|_{p ; \mathbb{R}^{n}} \leq & c_{0}\left[|h|^{1 / \alpha^{\prime}}\left\|\left(\int_{\rho}^{\infty}\left|F^{\prime}\right|^{\alpha}\right)^{1 / \alpha} \rho^{(n-1) / p}\right\|_{p ;(0,3 M|h|)}\right. \\
& +|h|^{M}\left(\left\|F^{\prime}(\rho) \rho^{1-M+(n-1) / p}\right\|_{p ;(M|h|, \infty)}\right. \\
& \left.\left.+\left\|F^{(M)}(\rho) \rho^{(n-1) / p}\right\|_{p ;(M|h|, \infty)}\right)\right] .
\end{aligned}
$$

(iii) Recall that (16) holds if $F$ has a compact support.

Proof of Lemma 4.2. Take $\xi>0$ and put

$$
B(\xi)=\left\{x \in \mathbb{R}^{n}:|x| \leq \xi\right\} \quad \text { and } \quad B^{\mathrm{c}}(\xi)=\mathbb{R}^{n} \backslash B(\xi) .
$$

Let $h \in \mathbb{R}^{n} \backslash\{0\}$. Then

$$
\left\|\Delta_{h}^{M} f\right\|_{p ; \mathbb{R}^{n}} \leq\left\|\Delta_{h}^{M} f\right\|_{p ; B(2 M|h|)}+\left\|\Delta_{h}^{M} f\right\|_{p ; B^{c}(2 M|h|)} .
$$

By (24),

$$
\left\|\Delta_{h}^{M} f\right\|_{p ; B^{c}(2 M|h|)} \lesssim|h|^{M} \sum_{k=1}^{M}\left\|F^{(k)}(\rho) \rho^{k-M+(n-1) / p}\right\|_{p ;(M|h|, \infty)}
$$

and so it is sufficient to show that

$$
\left\|\Delta_{h}^{M} f\right\|_{p ; B(2 M|h|)} \lesssim|h|^{1 / \alpha^{\prime}}\left\|\left(\int_{\rho}^{\infty}\left|F^{\prime}\right|^{\alpha}\right)^{1 / \alpha} \rho^{(n-1) / p}\right\|_{p ;(0,3 M|h|)}
$$

First assume that $M=1$. Let $\alpha \in[1, \infty)$. Then the Hölder inequality and the triangle inequality give

$$
\begin{aligned}
& \left|\Delta_{h} f(x)\right|=|F(|x+h|)-F(|x|)|=\left|\int_{|x|}^{|x+h|} F^{\prime}(\tau) \mathrm{d} \tau\right| \\
& \quad \leq|| x+\left.\left.h|-| x||^{1 / \alpha^{\prime}}\left|\int_{|x|}^{|x+h|}\right| F^{\prime}(\tau)\right|^{\alpha} \mathrm{d} \tau\right|^{1 / \alpha} \leq\left.\left.|h|^{1 / \alpha^{\prime}}\left|\int_{|x|}^{|x+h|}\right| F^{\prime}(\tau)\right|^{\alpha} \mathrm{d} \tau\right|^{1 / \alpha} .
\end{aligned}
$$


Consequently, for any $K \geq 2$,

$$
\begin{aligned}
& \left\|\Delta_{h} f\right\|_{p ; B(K|h|)} \leq\left\|\Delta_{h} f\right\|_{p ; B(K|h|) \cap\{|x| \leq|x+h|\}}+\left\|\Delta_{h} f\right\|_{p ; B(K|h|) \cap\{|x|>|x+h|\}} \\
& \leq|h|^{1 / \alpha^{\prime}}\left(\left\|\left(\int_{|x|}^{\infty}\left|F^{\prime}\right|^{\alpha}\right)^{1 / \alpha}\right\|_{p ; B(K|h|)}+\left\|\left(\int_{|x+h|}^{\infty}\left|F^{\prime}\right|^{\alpha}\right)^{1 / \alpha}\right\|_{p ; B(K|h|)}\right) .
\end{aligned}
$$

Thus, using the change of variables $y=x+h$ and then spherical coordinates, we arrive at

$$
\begin{aligned}
& \left\|\Delta_{h} f\right\|_{p ; B(K|h|)} \\
& \quad \leq|h|^{1 / \alpha^{\prime}}\left(\left\|\left(\int_{|x|}^{\infty}\left|F^{\prime}\right|^{\alpha}\right)^{1 / \alpha}\right\|_{p ; B(K|h|)}+\left\|\left(\int_{|y|}^{\infty}\left|F^{\prime}\right|^{\alpha}\right)^{1 / \alpha}\right\|_{p ; B(K|h|+|h|)}\right) \\
& \quad \lesssim|h|^{1 / \alpha^{\prime}}\left\|\left(\int_{\rho}^{\infty}\left|F^{\prime}\right|^{\alpha}\right)^{1 / \alpha} \rho^{(n-1) / p}\right\|_{p ;(0, K|h|+|h|)} \cdot
\end{aligned}
$$

Putting here $K=2$, we see that (59) with $M=1$ is satisfied.

Assume now that $M>1$. Then $\Delta_{h}^{M} f=\Delta_{h}^{M-1}\left(\Delta_{h} f\right)$. Applying (21) (with $M-1$ instead of $M$ ), the triangle inequality, the change of variables $y=x+k h$, and then (60) with $K=3 M-1$, we obtain

$$
\begin{aligned}
& \left\|\Delta_{h}^{M} f(x)\right\|_{p ; B(2 M|h|)} \leq \sum_{k=0}^{M-1}\left(\begin{array}{c}
M-1 \\
k
\end{array}\right)\left\|\Delta_{h} f(x+k h)\right\|_{p ; B(2 M|h|)} \\
& \quad \lesssim\left\|\Delta_{h} f(y)\right\|_{p ; B((3 M-1)|h|)} \lesssim|h|^{1 / \alpha^{\prime}}\left\|\left(\int_{\rho}^{\infty}\left|F^{\prime}\right|^{\alpha}\right)^{1 / \alpha} \rho^{(n-1) / p}\right\|_{p ;(0,3 M|h|)}
\end{aligned}
$$

and (59) is verified.

Lemma 4.4. Suppose that (53) holds. Let $F$ be given by (55). Then there is a positive constant $c=c(\alpha, \theta, M, n, p)$ such that

$$
\begin{aligned}
\left\|\left(\int_{\rho}^{\infty}\left|F^{\prime}\right|^{\alpha}\right)^{1 / \alpha} \rho^{(n-1) / p}\right\|_{p ;(0, t)} & \leq c R^{-1 / \alpha^{\prime}} \ell(R)^{-1-\theta} t^{n / p} \\
\left\|F^{\prime}(\rho) \rho^{1-M+(n-1) / p}\right\|_{p ;(t, \infty)} & \leq c R^{-M+n / p} \ell(R)^{-1-\theta} \\
\left\|F^{(M)}(\rho) \rho^{(n-1) / p}\right\|_{p ;(t, \infty)} & \leq c R^{-M+n / p} \ell(R)^{-1-\theta}
\end{aligned}
$$

for all $t \in(0, R]$, and

$$
\begin{aligned}
\left\|\left(\int_{\rho}^{\infty}\left|F^{\prime}\right|^{\alpha}\right)^{1 / \alpha} \rho^{(n-1) / p}\right\|_{p ;(0, t)} & \leq c \min \left\{t^{n / p-1 / \alpha^{\prime}} \ell(t)^{-1-\theta}, 1\right\} \\
\left\|F^{\prime}(\rho) \rho^{1-M+(n-1) / p}\right\|_{p ;(t, \infty)} & \leq c t^{-M+n / p} \ell(t)^{-1-\theta} \chi_{(R, 1)}(t), \\
\left\|F^{(M)}(\rho) \rho^{(n-1) / p}\right\|_{p ;(t, \infty)} & \leq c t^{-M+n / p} \ell(t)^{-1-\theta} \chi_{(R, 1)}(t)
\end{aligned}
$$

for all $t \in(R, \infty)$. 
Proof. Observe that

$$
\left|F^{(m)}(\rho)\right| \lesssim \rho^{-m} \ell(\rho)^{-1-\theta} \chi_{(R, 1)}(\rho) \text { for all } \rho>0 \text { and for } m \in\{1, M\} .
$$

Indeed, the estimate is obvious when $M=1$ (cf. (55)). If $M \geq 2$ and $m=1$, then, by $(55),(56)$, we obtain, for all $\rho>0$,

$$
\begin{aligned}
\left|F^{\prime}(\rho)\right| & =\psi_{R}(\rho) \int_{\rho}^{\infty}(\tau-\rho)^{M-2} \tau^{-M} \ell(\tau)^{-1-\theta} \chi_{(R, 1)}(\tau) \mathrm{d} \tau \\
& \leq \chi_{(R, 1)}(\rho) \int_{\rho}^{\infty} \tau^{-2} \ell(\tau)^{-1-\theta} \mathrm{d} \tau \lesssim \rho^{-1} \ell(\rho)^{-1-\theta} \chi_{(R, 1)}(\rho) .
\end{aligned}
$$

If $M \geq 2$ and $m=M$, then, using (55)-(57), we arrive at

$$
\begin{aligned}
& \left|F^{(M)}(\rho)\right|=\left|\frac{\mathrm{d}^{M-1}}{\mathrm{~d} \rho^{M-1}}\left(\psi_{R}(\rho) \int_{\rho}^{\infty}(\tau-\rho)^{M-2} \tau^{-M} \ell(\tau)^{-1-\theta} \chi_{(R, 1)}(\tau) \mathrm{d} \tau\right)\right| \\
& =\left|\sum_{k=0}^{M-1}\left(\begin{array}{c}
M-1 \\
k
\end{array}\right) \frac{\mathrm{d}^{k}}{\mathrm{~d} \rho^{k}} \psi_{R}(\rho) \frac{\mathrm{d}^{M-1-k}}{\mathrm{~d} \rho^{M-1-k}}\left(\int_{\rho}^{\infty}(\tau-\rho)^{M-2} \tau^{-M} \ell(\tau)^{-1-\theta} \chi_{(R, 1)}(\tau) \mathrm{d} \tau\right)\right| \\
& \lesssim \rho^{-M} \ell(\rho)^{-1-\theta} \chi_{(R, 1)}(\rho) \\
& \quad+\sum_{k=1}^{M-1} R^{-k} \chi_{(R, 2 R)}(\rho) \int_{\rho}^{\infty}(\tau-\rho)^{k-1} \tau^{-M} \ell(\tau)^{-1-\theta} \chi_{(R, 1)}(\tau) \mathrm{d} \tau \\
& \lesssim \rho^{-M} \ell(\rho)^{-1-\theta} \chi_{(R, 1)}(\rho)+\sum_{k=1}^{M-1} \rho^{-k} \chi_{(R, 2 R)}(\rho) \int_{\rho}^{\infty} \tau^{k-1-M} \ell(\tau)^{-1-\theta} \chi_{(R, 1)}(\tau) \mathrm{d} \tau \\
& \approx \rho^{-M} \ell(\rho)^{-1-\theta} \chi_{(R, 1)}(\rho), \quad \rho>0,
\end{aligned}
$$

and $(67)$ is verified.

Since $\alpha \in(1, \infty),(67)$ implies that

$$
\left(\int_{\rho}^{\infty}\left|F^{\prime}(\tau)\right|^{\alpha} \mathrm{d} \tau\right)^{1 / \alpha} \lesssim \begin{cases}0 & \text { if } \rho \in[1, \infty), \\ \rho^{-1 / \alpha^{\prime}} \ell(\rho)^{-1-\theta} & \text { if } \rho \in[R, 1), \\ R^{-1 / \alpha^{\prime}} \ell(R)^{-1-\theta} & \text { if } \rho \in[0, R)\end{cases}
$$

Assume now that $t \in(0, R]$. Then, by $(68)$,

$$
\begin{aligned}
\left\|\left(\int_{\rho}^{\infty}\left|F^{\prime}\right|^{\alpha}\right)^{1 / \alpha} \rho^{(n-1) / p}\right\|_{p ;(0, t)} & \lesssim R^{-1 / \alpha^{\prime}} \ell(R)^{-1-\theta}\left\|\rho^{(n-1) / p}\right\|_{p ;(0, t)} \\
& \approx R^{-1 / \alpha^{\prime}} \ell(R)^{-1-\theta} t^{n / p}
\end{aligned}
$$

which gives (61). Furthermore, since $M>n / p$ (cf. (53)), (67) yields

$$
\begin{aligned}
\left\|F^{\prime}(\rho) \rho^{1-M+(n-1) / p}\right\|_{p ;(t, \infty)} & =\left\|F^{\prime}(\rho) \rho^{1-M+(n-1) / p}\right\|_{p ;(R, 1)} \\
= & \left\|\rho^{-M+n / p-1 / p} \ell(\rho)^{-1-\theta}\right\|_{p ;(R, 1)} \lesssim R^{-M+n / p} \ell(R)^{-1-\theta}
\end{aligned}
$$


and (62) is proved. Using (67) and the inequality $n / p-M<0$, we get

$$
\left\|F^{(M)}(\rho) \rho^{(n-1) / p}\right\|_{p ;(t, \infty)}=\left\|\rho^{-M} \ell(\rho)^{-1-\theta} \rho^{(n-1) / p}\right\|_{p ;(R, 1)} \lesssim R^{-M+n / p} \ell(R)^{-1-\theta}
$$

and (63) is verified.

Suppose that $t \in(R, 1)$. Then

$$
\begin{aligned}
& \left\|\left(\int_{\rho}^{\infty}\left|F^{\prime}\right|^{\alpha}\right)^{1 / \alpha} \rho^{(n-1) / p}\right\|_{p ;(0, t)} \\
& \quad \leq\left\|\left(\int_{\rho}^{\infty}\left|F^{\prime}\right|^{\alpha}\right)^{1 / \alpha} \rho^{(n-1) / p}\right\|_{p ;(0, R)}+\left\|\left(\int_{\rho}^{\infty}\left|F^{\prime}\right|^{\alpha}\right)^{1 / \alpha} \rho^{(n-1) / p}\right\|_{p ;[R, t)} .
\end{aligned}
$$

By $(61)$,

$$
\left\|\left(\int_{\rho}^{\infty}\left|F^{\prime}\right|^{\alpha}\right)^{1 / \alpha} \rho^{(n-1) / p}\right\|_{p ;(0, R)} \lesssim R^{n / p-1 / \alpha^{\prime}} \ell(R)^{-1-\theta} .
$$

Moreover, applying (68) and the condition $n / p-1 / \alpha^{\prime}>0$ (cf. (53)), we obtain

$$
\begin{aligned}
\left\|\left(\int_{\rho}^{\infty}\left|F^{\prime}\right|^{\alpha}\right)^{1 / \alpha} \rho^{(n-1) / p}\right\|_{p ;[R, t)} & \lesssim\left\|\rho^{-1 / \alpha^{\prime}} \ell(\rho)^{-1-\theta} \rho^{(n-1) / p}\right\|_{p ;[R, t)} \\
& \lesssim\left\|\rho^{n / p-1 / \alpha^{\prime}-1 / p} \ell(\rho)^{-1-\theta}\right\|_{p ;(0, t)} \approx t^{n / p-1 / \alpha^{\prime}} \ell(t)^{-1-\theta} .
\end{aligned}
$$

The estimate (64) is a consequence of (69)-(71). Furthermore, (67) and the inequality $n / p-M<0$ imply that

$$
\begin{aligned}
\left\|F^{\prime}(\rho) \rho^{1-M+(n-1) / p}\right\|_{p ;(t, \infty)} & =\left\|F^{\prime}(\rho) \rho^{1-M+(n-1) / p}\right\|_{p ;(t, 1)} \\
& \lesssim\left\|\rho^{-M+n / p-1 / p} \ell(\rho)^{-1-\theta}\right\|_{p ;(t, 1)} \lesssim t^{-M+n / p} \ell(t)^{-1-\theta}
\end{aligned}
$$

and (65) follows. The estimate (66) can be proved similarly as (63).

Finally, let $t \in[1, \infty)$. Then, since $\operatorname{supp} F=[0,1]$,

$$
\left\|\left(\int_{\rho}^{\infty}\left|F^{\prime}\right|^{\alpha}\right)^{1 / \alpha} \rho^{(n-1) / p}\right\|_{p ;(0, t)}=\left\|\left(\int_{\rho}^{\infty}\left|F^{\prime}\right|^{\alpha}\right)^{1 / \alpha} \rho^{(n-1) / p}\right\|_{p ;(0,1)} \lesssim 1
$$

(cf. (69)-(71)) and

$$
\left\|F^{\prime}(\rho) \rho^{1-M+(n-1) / p}\right\|_{p ;(t, \infty)}=0, \quad\left\|F^{(M)}(\rho) \rho^{(n-1) / p}\right\|_{p ;(t, \infty)}=0 .
$$

Thus (64), (65), and (66) again hold.

Lemma 4.5. Suppose that (53) holds. Let $f(x)=F(|x|)=F_{R}(|x|), x \in \mathbb{R}^{n}$, where the function $F$ is given by (55). Then there is a positive constant $C=C(\alpha, \theta, M, n, p)$ such that, for all $\tau \in(0,1)$,

$$
\omega_{M}(f, \tau)_{p} \leq C\left[R^{-1 / \alpha^{\prime}} \ell(R)^{-1-\theta} \tau^{n / p+1 / \alpha^{\prime}} \chi_{(0, R]}(\tau)+\tau^{n / p} \ell(\tau)^{-1-\theta} \chi_{(R, 1)}(\tau)\right]
$$


Proof. Let $h \in \mathbb{R}^{n}, 0<|h|<1$. By (58),

$$
\left\|\Delta_{h}^{M} f\right\|_{p ; \mathbb{R}^{n}} \lesssim N_{1}(|h|)+N_{2}(|h|),
$$

where

$$
\begin{aligned}
& N_{1}(\tau):=\tau^{1 / \alpha^{\prime}}\left\|\left(\int_{\rho}^{\infty}\left|F^{\prime}\right|^{\alpha}\right)^{1 / \alpha} \rho^{(n-1) / p}\right\|_{p ;(0,3 M \tau)}, \\
& N_{2}(\tau):=\tau^{M}\left(\left\|F^{\prime}(\rho) \rho^{1-M+(n-1) / p}\right\|_{p ;(M \tau, \infty)}+\left\|F^{(M)}(\rho) \rho^{(n-1) / p}\right\|_{p ;(M \tau, \infty)}\right)
\end{aligned}
$$

for $\tau \in(0,1)$. Moreover, by Lemma 4.4,

$$
N_{1}(\tau) \lesssim\left\{\begin{array}{lll}
R^{-1 / \alpha^{\prime}} \ell(R)^{-1-\theta} \tau^{n / p+1 / \alpha^{\prime}} & \text { if } & 0 \leq \tau \leq R /(3 M), \\
\tau^{n / p} \ell(\tau)^{-1-\theta} & \text { if } & R /(3 M)<\tau<1
\end{array}\right.
$$

and

$$
N_{2}(\tau) \lesssim \begin{cases}R^{-M+n / p} \ell(R)^{-1-\theta} \tau^{M} & \text { if } \quad 0 \leq \tau \leq R / M \\ \tau^{n / p} \ell(\tau)^{-1-\theta} & \text { if } \quad R / M<\tau<1\end{cases}
$$

These estimates immediately imply that

$$
N_{1}(\tau)+N_{2}(\tau) \lesssim \tau^{n / p} \ell(\tau)^{-1-\theta} \quad \text { for all } \tau \in(R, 1)
$$

Observe that

$$
N_{1}(\tau)+N_{2}(\tau) \lesssim R^{-1 / \alpha^{\prime}} \ell(R)^{-1-\theta} \tau^{n / p+1 / \alpha^{\prime}} \quad \text { for all } \tau \in(0, R]
$$

Indeed, it is clear from (74), (75) when $R /(3 M)<\tau \leq R$. If $\tau \in(0, R /(3 M))$, then (77) follows again from (74), (75) due to the inequality $n / p+1 / \alpha^{\prime}<M$ (cf. (53)).

The estimate (72) is a consequence of (73), (76), and (77) and the fact that the function $t \mapsto R^{-1 / \alpha^{\prime}} \ell(R)^{-1-\theta} t^{n / p+1 / \alpha^{\prime}} \chi_{(0, R)}(t)+t^{n / p} \ell(t)^{-1-\theta} \chi_{(R, 1)}(t)$ is equivalent to an increasing function on $(0,1)$.

Now, we are able to estimate (from above) the norm of the test function $f=f_{R}$ in the space $B_{p, r}^{n / p, \delta+1 / r^{\prime}}$.

Lemma 4.6. Suppose that (53) holds and $1 \leq r \leq \infty$. Let $f(x)=F(|x|)=F_{R}(|x|)$, $x \in \mathbb{R}^{n}$, where the function $F$ is given by (55). Then there is a positive constant $\bar{c}$ independent of $R$ such that, for all $R \in(0,1 / 2)$,

$$
\|f\|_{B_{p, r}^{n / p, \delta+1 / r^{\prime}}} \leq \bar{c} \ell(R)^{\delta-\theta} .
$$


Proof. One can easily derive from (55) that, for $\rho \in[0, \infty)$,

$$
|F(\rho)| \lesssim \int_{(\rho, \infty) \cap(R, 1)} \tau^{-1} \ell(\tau)^{-1-\theta} \mathrm{d} \tau .
$$

Consequently,

$$
|F(\rho)| \lesssim\left\{\begin{array}{lll}
0 & \text { if } \quad \rho \in[1, \infty), \\
\ell(\rho)^{-\theta} & \text { if } \quad \rho \in[R, 1), \\
\ell(R)^{-\theta} & \text { if } \quad \rho \in[0, R) .
\end{array}\right.
$$

Thus, using (54) and spherical coordinates, we obtain

$$
\begin{aligned}
\|f\|_{p} & =s_{n}^{1 / p}\left\|F(\rho) \rho^{(n-1) / p}\right\|_{p ;(0, \infty)} \\
& \lesssim\left\|F(\rho) \rho^{(n-1) / p}\right\|_{p ;(0, R)}+\left\|F(\rho) \rho^{(n-1) / p}\right\|_{p ;(R, 1)} \\
& \lesssim \ell(R)^{-\theta}\left\|\rho^{(n-1) / p}\right\|_{p ;(0, R)}+\left\|\rho^{(n-1) / p} \ell(\rho)^{-\theta}\right\|_{p ;(R, 1)} \\
& \lesssim R^{n / p} \ell(R)^{-\theta}+\left\|\rho^{(n-1) / p} \ell(\rho)^{-\theta}\right\|_{p ;(0,1)} \\
& \approx R^{n / p} \ell(R)^{-\theta}+1 \lesssim 1 \quad \text { for all } R \in(0,1 / 2) .
\end{aligned}
$$

Moreover, applying Lemma 4.5 and making use of (53), we arrive at

$$
\begin{aligned}
& \| t^{-n / p-1 / r} \ell(t)^{\delta+1 / r^{\prime}} \omega_{M}(f, t)_{p} \|_{r ;(0,1)} \\
& \lesssim R^{-1 / \alpha^{\prime}} \ell(R)^{-1-\theta}\left\|t^{-n / p-1 / r} \ell(t)^{\delta+1 / r^{\prime}} t^{n / p+1 / \alpha^{\prime}}\right\|_{r ;(0, R)} \\
&+\left\|t^{-n / p-1 / r} \ell(t)^{\delta+1 / r^{\prime}} t^{n / p} \ell(t)^{-1-\theta}\right\|_{r ;(R, 1)} \\
& \lesssim R^{-1 / \alpha^{\prime}} \ell(R)^{-1-\theta} R^{1 / \alpha^{\prime}} \ell(R)^{\delta+1 / r^{\prime}}+\ell(R)^{\delta-\theta} \\
& \lesssim \ell(R)^{\delta-\theta} \quad \text { for all } R \in(0,1 / 2) .
\end{aligned}
$$

Together with (79), this implies that

$$
\|f\|_{B_{p, r}^{n / p, \delta+1 / r^{\prime}}} \lesssim \ell(R)^{\delta-\theta} \quad \text { for all } R \in(0,1 / 2) .
$$

The next lemma gives an estimate (from below) of the left-hand side of inequality (6) with an appropriate choice of a function $f$.

Lemma 4.7. Suppose that the assumptions of Theorem 2.3 are satisfied and that (53) holds. Let $f(x)=F(|x|)=F_{R}(|x|), x \in \mathbb{R}^{n}$, where the function $F_{R}$ is given by $(55)$. Let $\kappa \in \mathfrak{M}^{+}(0,1 ; \downarrow)$ and $1 \leq s \leq \infty$. Then there is a positive constant $\bar{C}$ independent of $R$ such that

$$
\left\|t^{-1 / s} \ell(t)^{\delta-1 / s} \kappa(t) f^{*}(t)\right\|_{s ;(0,1)} \geq \bar{C} \ell(R)^{\delta-\theta} \kappa\left(\beta_{n} R^{n}\right)
$$

for all $R \in\left(0, R_{0}\right)$, where $R_{0}=\min \left\{4^{-2}, \beta_{n}^{-1 / n}\right\}$. 
Proof. Observe that for any $x \in \mathbb{R}^{n},|x|<R$, where $R \in\left(0,4^{-2}\right)$,

$$
f(x) \gtrsim \ell(R)^{-\theta} \text {. }
$$

Indeed, if $M=1$, then (54), (55) and the condition $\theta<0$ (cf. (53)) imply that

$$
f(x) \geq \int_{2 R}^{1} t^{-1} \ell(t)^{-1-\theta} \mathrm{d} t \approx \ell(R)^{-\theta} .
$$

If $M \geq 2$, then, by (54), (55), (56) and the condition $\theta<0$,

$$
\begin{aligned}
f(x) & \geq \int_{|x|}^{\infty} \chi_{(2 R, \infty)}(t) \int_{t}^{\infty}(\tau-t)^{M-2} \tau^{-M} \ell(\tau)^{-1-\theta} \chi_{(R, 1)}(\tau) \mathrm{d} \tau \mathrm{d} t \\
& \geq \int_{2 R}^{1 / 4} \int_{2 t}^{\infty}(\tau-\tau / 2)^{M-2} \tau^{-M} \ell(\tau)^{-1-\theta} \chi_{(R, 1)}(\tau) \mathrm{d} \tau \mathrm{d} t \\
& \approx \int_{2 R}^{1 / 4} \int_{2 t}^{1} \tau^{-2} \ell(\tau)^{-1-\theta} \mathrm{d} \tau \mathrm{d} t \approx \ell(R)^{-\theta}
\end{aligned}
$$

and (81) again follows.

The estimate (81) yields

$$
f^{*}(t) \gtrsim \ell(R)^{-\theta} \chi_{\left(0, \beta_{n} R^{n}\right)}(t) \text { for all } t \in(0, \infty) \text { and } R \in\left(0,4^{-2}\right) .
$$

Consequently, for all $R \in\left(0, R_{0}\right)$,

$$
\begin{aligned}
\left\|t^{-1 / s} \ell(t)^{\delta-1 / s} \kappa(t) f^{*}(t)\right\|_{s ;(0,1)} & \gtrsim \ell(R)^{-\theta}\left\|t^{-1 / s} \ell(t)^{\delta-1 / s} \kappa(t)\right\|_{s ;\left(0, \beta_{n} R^{n}\right)} \\
& \gtrsim \ell(R)^{-\theta} \kappa\left(\beta_{n} R^{n}\right)\left\|t^{-1 / s} \ell(t)^{\delta-1 / s}\right\|_{s ;\left(0, \beta_{n} R^{n}\right)} \\
& \approx \ell(R)^{-\theta} \kappa\left(\beta_{n} R^{n}\right) \ell(R)^{\delta}=\ell(R)^{\delta-\theta} \kappa\left(\beta_{n} R^{n}\right),
\end{aligned}
$$

and (80) is verified.

Proof of the implication (i) $\Rightarrow$ (ii). Let $f=f_{R}, R \in\left(0, R_{0}\right)$, be the function from Lemma 4.7. Then inequality (6) and estimates (78) and (80) imply that

$$
\bar{C} \ell(R)^{\delta-\theta} \kappa\left(\beta_{n} R^{n}\right) \leq C \bar{c} \ell(R)^{\delta-\theta} \quad \text { for all } R \in\left(0, R_{0}\right) .
$$

Consequently,

$$
\kappa\left(\beta_{n} R^{n}\right) \leq C \bar{c} / \bar{C} \quad \text { for all } R \in\left(0, R_{0}\right)
$$

and (ii) follows.

Remark 4.8. If $n / p \notin \mathbb{N}$, one can choose $M \in(n / p, 1+n / p)$ in (53). Then, instead of the function $F$ given by (55), one can use the function $F$ from (27) with

$$
g(t)=g_{R}(t)=t^{-M} \ell(t)^{-1-\theta} \chi_{(R, 1)}(t), \quad t \in \mathbb{R},
$$


where $R \in(0,1)$ is a fixed number. Note also that such $F$, instead of $(67)$ with $m=1$, satisfies

$$
\left|F^{\prime}(\rho)\right| \lesssim\left\{\begin{array}{lll}
0 & \text { if } & \rho \in(1, \infty), \\
\rho^{-1} \ell(\rho)^{-1-\theta} & \text { if } \quad \rho \in(R, 1), \\
R^{-1} \ell(R)^{-1-\theta} & \text { if } \quad \rho \in(0, R) .
\end{array}\right.
$$

Nevertheless, all lemmas of section 4 remain true. However, this is not the case when $n / p \in \mathbb{N}$. Then one has to take $M=1+n / p$ in (53) and one can show that all the estimates in Lemma 4.4 are satisfied (with $F$ from (27) and $g$ given by (82)) except (62). Instead of (62), one can only derive that

$$
\left\|F^{\prime}(\rho) \rho^{1-M+(n-1) / p}\right\|_{p ;(t, \infty)} \lesssim t^{-M+n / p} \ell(R)^{-1-\theta}
$$

for all $t \in(0, R]$. This causes that the test function $f$ corresponding to $F$ can be used to prove the sharpness of Theorem 2.3 only if $\delta$ satisfies the additional restriction $\delta<-1$. This is why the cut-off function $\psi_{R}$ was used in (55) to define $F$.

\section{Appendix}

The aim of this section is to give proofs of assertions mentioned in Remarks 2.5 (i) and (ii).

First, we have promised to prove lower estimates for the growth envelope functions, that is, to prove that (cf. (9))

$$
\mathcal{E}_{G} \mid B_{p, r}^{\sigma, \alpha}(t) \gtrsim t^{-1 / q} \ell^{-\alpha}(t) \quad \text { for all } t \in(0, \varepsilon)
$$

provided that (10) is satisfied, and that (cf. (11))

$$
\mathcal{E}_{G} \mid B_{p, r}^{n / p, \delta+1 / r^{\prime}}(t) \gtrsim \ell^{-\delta}(t) \quad \text { for all } t \in(0, \varepsilon)
$$

provided that (12) holds.

Moreover, we have promised to show that the inequality

$$
\left\|t^{1 / q-1 / v} \ell^{\alpha}(t) f^{*}(t)\right\|_{v ;(0,1)} \lesssim\|f\|_{B_{p, r}^{\sigma, \alpha}}
$$

does not hold for all $f \in B_{p, r}^{\sigma, \alpha}\left(\mathbb{R}^{n}\right)$ if (10) is satisfied and $0<v<r$, and that the inequality

$$
\left\|t^{-1 / v} \ell^{\delta-1 / v}(t) f^{*}(t)\right\|_{v ;(0,1)} \lesssim\|f\|_{B_{p, r}^{n / p, \delta+1 / r^{\prime}}}
$$

does not hold for all $f \in B_{p, r}^{n / p, \delta+1 / r^{\prime}}\left(\mathbb{R}^{n}\right)$ if $(12)$ is satisfied and $0<v<r$.

(i) To verify (83), take $f=f_{R}$ from Lemma 3.8. Then, cf. (52),

$$
f_{R}^{*}(t) \geq c R^{\gamma} \chi_{\left(0, \beta_{n} R^{n}\right)}(t) \quad \text { for all } t \in(0, \infty) \text { and every } R \in\left(0, R_{1}\right) .
$$


In particular,

$$
f_{R}^{*}\left(\beta_{n} R^{n} / 2\right) \geq c R^{\gamma} \quad \text { for every } R \in\left(0, R_{1}\right) .
$$

Put $h_{R}=f_{R} /\left\|f_{R}\right\|_{B_{p, r}^{\sigma, \alpha}}$. Then, by (87) and (45),

$$
\begin{aligned}
h_{R}^{*}\left(\beta_{n} R^{n} / 2\right) & =\frac{f_{R}^{*}\left(\beta_{n} R^{n} / 2\right)}{\left\|f_{R}\right\|_{B_{p}^{\sigma, r}}^{\sigma, \alpha}} \geq \frac{c R^{\gamma}}{\bar{c} R^{\gamma-\sigma+n / p} \ell^{\alpha}(R)} \\
& \approx R^{\sigma-n / p} \ell^{-\alpha}(R)=R^{-n / q} \ell^{-\alpha}(R) \\
& \approx\left(\beta_{n} R^{n} / 2\right)^{-1 / q} \ell^{-\alpha}\left(\beta_{n} R^{n} / 2\right) \text { for all } R \in\left(0, R_{1}\right) .
\end{aligned}
$$

Thus, taking $\varepsilon=\beta_{n} R_{1}^{n} / 2$, we can see that

$$
h_{R}^{*}(t) \gtrsim t^{-1 / q} \ell^{-\alpha}(t) \text { for all } t \in(0, \varepsilon),
$$

and (83) follows since $\left\|h_{R}\right\|_{B_{p, r}^{\sigma, \alpha}}=1$ for all $R \in(0,1)$.

(ii) To verify (84), we take $f=f_{R}$ from Lemma 4.7 and apply the same arguments.

(iii) To prove that (85) does not hold on $B_{p, r}^{\sigma, \alpha}\left(\mathbb{R}^{n}\right)$ if (10) is satisfied and $0<v<r$, define $f$ by

$$
f(x)=F(|x|), \quad x \in \mathbb{R}^{n},
$$

with

$$
F(\rho)=\int_{\rho}^{\infty}(t-\rho)^{M-1} \chi_{\left(0,2 \beta_{n}^{-1 / n}\right)}(t) t^{-n / q-M} \ell(t)^{-\alpha-1 / v} \mathrm{~d} t,
$$

where $\rho \in[0, \infty), M \in \mathbb{N}, M \in[n / p, 1+n / p)$. Then

$$
f(x) \approx|x|^{-n / q} \ell(|x|)^{-\alpha-1 / v} \quad \text { if } \quad|x|<\beta_{n}^{-1 / n}
$$

and

$$
f^{*}(t) \approx t^{-1 / q} \ell(t)^{-\alpha-1 / v} \quad \text { for all } t \in(0,1) .
$$

Consequently, the left-hand side of (85) is infinite, since

$$
\left\|t^{1 / q-1 / v} \ell(t)^{\alpha} t^{-1 / q} \ell(t)^{-\alpha-1 / v}\right\|_{v ;(0,1)}=\left\|t^{-1 / v} \ell(t)^{-1 / v}\right\|_{v ;(0,1)}=\infty .
$$

Thus, it is sufficient to prove that the right-hand side of (85) is finite, that is,

$$
\|f\|_{B_{p, r}^{\sigma, \alpha}}<\infty
$$


Let $h \in \mathbb{R}^{n}, 0<|h|<(3 M)^{-1}$. Then, using Remarks 3.4(i), (iii) and the identity $\sigma=n / p-n / q$, we obtain

$$
\begin{aligned}
\left\|\Delta_{h}^{M} f\right\|_{p} \lesssim & \left\|F(\rho) \rho^{(n-1) / p}\right\|_{p ;(0,3 M|h|)}+|h|^{M}\left\|F^{(M)}(\rho) \rho^{(n-1) / p}\right\|_{p ;(M|h|, \infty)} \\
\lesssim & \left\|\rho^{-n / q} \ell(\rho)^{-\alpha-1 / v} \rho^{(n-1) / p}\right\|_{p ;(0,3 M|h|)} \\
& \quad+|h|^{M}\left\|\rho^{-n / q-M} \ell(\rho)^{-\alpha-1 / v} \rho^{(n-1) / p}\right\|_{p ;(M|h|, \infty)} \\
= & \left\|\rho^{\sigma-1 / p} \ell(\rho)^{-\alpha-1 / v}\right\|_{p ;(0,3 M|h|)} \\
& \quad+|h|^{M}\left\|\rho^{\sigma-M-1 / p} \ell(\rho)^{-\alpha-1 / v}\right\|_{p ;(M|h|, \infty)} \\
\approx & |h|^{\sigma} \ell(|h|)^{-\alpha-1 / v}+|h|^{M}|h|^{\sigma-M} \ell(|h|)^{-\alpha-1 / v} \\
\approx & |h|^{\sigma} \ell(|h|)^{-\alpha-1 / v} .
\end{aligned}
$$

Therefore,

$$
\omega_{M}(f, t)_{p} \lesssim t^{\sigma} \ell(t)^{-\alpha-1 / v} \quad \text { for all } t \in\left(0,(3 M)^{-1}\right),
$$

which, since $1 / r-1 / v<0$, implies that

$$
\left\|t^{-\sigma-1 / r} \ell^{\alpha}(t) \omega_{M}(f, t)_{p}\right\|_{r ;\left(0,(3 M)^{-1}\right)} \lesssim\left\|t^{-1 / r} \ell(t)^{-1 / v}\right\|_{r ;\left(0,(3 M)^{-1}\right)}<\infty .
$$

Moreover,

$$
\|f\|_{p ; \mathbb{R}^{n}}=\left\|f^{*}\right\|_{p ;(0, \infty)} \lesssim\left\|t^{-1 / q} \ell(t)^{-\alpha-1 / v}\right\|_{p ;\left(0,2^{n}\right)}<\infty .
$$

Together with (90), this implies (89).

(iv) To prove that (86) does not hold on $B_{p, r}^{n / p, \delta+1 / r^{\prime}}\left(\mathbb{R}^{n}\right)$ if (12) is satisfied and $0<v<r$, we define the function $f$ by $(88)$ with

$$
F(\rho)=\int_{\rho}^{\infty}(t-\rho)^{M-1} \chi_{\left(0,2 \beta_{n}^{-1 / n}\right)}(t) t^{-M} \ell(t)^{-\delta-1} \ell_{2}(t)^{-1 / v} \mathrm{~d} t
$$

where $\rho \in[0, \infty), M \in \mathbb{N}, M \in(n / p, 1+n / p]$. Then

$$
f(x) \approx \ell(|x|)^{-\delta} \ell_{2}(|x|)^{-1 / v} \quad \text { if } \quad|x|<\beta_{n}^{-1 / n}
$$

and

$$
f^{*}(t) \approx \ell(t)^{-\delta} \ell_{2}(t)^{-1 / v} \text { for all } t \in(0,1)
$$

Consequently, the left-hand side of (86) is infinite, since

$$
\left\|t^{-1 / v} \ell^{\delta-1 / v}(t) f^{*}(t)\right\|_{v ;(0,1)} \approx\left\|t^{-1 / v} \ell^{-1 / v}(t) \ell_{2}(t)^{-1 / v}\right\|_{v ;(0,1)}=\infty .
$$


Thus, it is sufficient to verify that the right-hand side of (86) is finite, that is,

$$
\|f\|_{B_{p, r}^{n / p, \delta+1 / r^{\prime}}}<\infty .
$$

The relation (91) implies that

$$
F^{\prime}(\rho)=0 \quad \text { if } \quad \rho \in\left(2 \beta_{n}^{-1 / n}, \infty\right),
$$

and

$$
\begin{aligned}
& \left|F^{\prime}(\rho)\right| \approx \int_{\rho}^{\infty}(t-\rho)^{M-2} \chi_{\left(0,2 \beta_{n}^{-1 / n}\right)}(t) t^{-M} \ell(t)^{-\delta-1} \ell_{2}(t)^{-1 / v} \mathrm{~d} t \\
& \lesssim \int_{\rho}^{\infty} t^{-2} \ell(t)^{-\delta-1} \ell_{2}(t)^{-1 / v} \mathrm{~d} t \approx \rho^{-1} \ell(\rho)^{-\delta-1} \ell_{2}(\rho)^{-1 / v} \quad \text { if } \rho \in\left(0,2 \beta_{n}^{-1 / n}\right) .
\end{aligned}
$$

Hence, if $\alpha>1$ and $\rho \in(0,1)$, then

$$
\left(\int_{\rho}^{\infty}\left|F^{\prime}(t)\right|^{\alpha} \mathrm{d} t\right)^{1 / \alpha} \lesssim \rho^{-1 / \alpha^{\prime}} \ell(\rho)^{-\delta-1} \ell_{2}(\rho)^{-1 / v}
$$

Using (58) with $\alpha>1$ such that $n / p-1 / \alpha^{\prime}>0$, we obtain, for all $h \in \mathbb{R}^{n}$, $0<|h|<(3 M)^{-1}$,

$$
\begin{aligned}
\left\|\Delta_{h}^{M} f\right\|_{p} \lesssim & |h|^{1 / \alpha^{\prime}}\left\|\left(\int_{\rho}^{\infty}\left|F^{\prime}\right|^{\alpha}\right)^{1 / \alpha} \rho^{(n-1) / p}\right\|_{p ;(0,3 M|h|)} \\
& +|h|^{M}\left(\left\|F^{\prime}(\rho) \rho^{1-M+(n-1) / p}\right\|_{p ;(M|h|, \infty)}\right. \\
& \left.+\left\|F^{(M)}(\rho) \rho^{(n-1) / p}\right\|_{p ;(M|h|, \infty)}\right) \\
\lesssim & |h|^{1 / \alpha^{\prime}}\left\|\rho^{-1 / \alpha^{\prime}+(n-1) / p} \ell(\rho)^{-\delta-1} \ell_{2}(\rho)^{-1 / v}\right\|_{p ;(0,3 M|h|)} \\
& +|h|^{M}\left\|\rho^{-M+(n-1) / p} \ell(\rho)^{-\delta-1} \ell_{2}(\rho)^{-1 / v}\right\|_{p ;(M|h|, \infty)} \\
\approx & |h|^{1 / \alpha^{\prime}}|h|^{-1 / \alpha^{\prime}+n / p} \ell(|h|)^{-\delta-1} \ell_{2}(|h|)^{-1 / v} \\
& +|h|^{M}|h|^{-M+n / p} \ell(|h|)^{-\delta-1} \ell_{2}(|h|)^{-1 / v} \\
\approx & |h|^{n / p} \ell(|h|)^{-\delta-1} \ell_{2}(|h|)^{-1 / v} .
\end{aligned}
$$

Thus, $\omega_{M}(f, t)_{p} \lesssim t^{n / p} \ell(t)^{-\delta-1} \ell_{2}(t)^{-1 / v}$ for all $t \in\left(0,(3 M)^{-1}\right)$. Since $1 / r-1 / v<0$, the last estimate yields

$$
\begin{aligned}
\| t^{-n / p-1 / r} \ell(t)^{\delta+1 / r^{\prime}} \omega_{M}(f, t)_{p} & \|_{r ;\left(0,(3 M)^{-1}\right)} \\
& \lesssim\left\|t^{-1 / r} \ell(t)^{-1 / r} \ell_{2}(t)^{-1 / v}\right\|_{r ;\left(0,(3 M)^{-1}\right)}<\infty .
\end{aligned}
$$

This, together with the estimate

$$
\|f\|_{p ; \mathbb{R}^{n}}=\left\|f^{*}\right\|_{p ;(0, \infty)} \lesssim\left\|\ell^{-\delta} \ell_{2}^{-1 / v}\right\|_{p ;\left(0,2^{n}\right)}<\infty,
$$

implies (92) 
Acknowledgements. We are indebted to Hans-Gerd Leopold from Friedrich Schiller University in Jena for useful discussions which we had with him during his visit to Prague and during the visits of the first author to Jena.

\section{References}

[1] R. A. Adams, Sobolev spaces, Academic Press, New York-London, 1975.

[2] C. Bennett and K. Rudnick, On Lorentz-Zygmund spaces, Dissertationes Math. (Rozprawy Mat.) 175 (1980), 67.

[3] C. Bennett and R. Sharpley, Interpolation of operators, Pure and Applied Mathematics, vol. 129, Academic Press Inc., Boston, MA, 1988.

[4] H. Brézis and S. Wainger, A note on limiting cases of Sobolev embeddings and convolution inequalities, Comm. Partial Differential Equations 5 (1980), no. 7, 773-789.

[5] A. Caetano and S. D. Moura, Local growth envelopes of spaces of generalized smoothness: the sub-critical case, to appear.

[6] Local growth envelopes of spaces of generalized smoothness: the critical case, to appear.

[7] D. E. Edmunds, P. Gurka, and B. Opic, Double exponential integrability of convolution operators in generalized Lorentz-Zygmund spaces, Indiana Univ. Math. J. 44 (1995), no. 1, 19-43.

[8] _ Double exponential integrability, Bessel potentials and embedding theorems, Studia Math. 115 (1995), no. 2, 151-181.

[9] Sharpness of embeddings in logarithmic Bessel-potential spaces, Proc. Roy. Soc. Edinburgh Sect. A 126 (1996), no. 5, 995-1009

[10] - On embeddings of logarithmic Bessel potential spaces, J. Funct. Anal. 146 (1997), no. 1, $116-150$.

[11] N Norms of embeddings of logarithmic Bessel potential spaces, Proc. Amer. Math. Soc. 126 (1998), no. 8, 2417-2425.

[12] _ Optimality of embeddings of logarithmic Bessel potential spaces, Q. J. Math. 51 (2000), no. $2,185-209$

[13] W. D. Evans, B. Opic, and L. Pick, Interpolation of operators on scales of generalized LorentzZygmund spaces, Math. Nachr. 182 (1996), 127-181.

[14] _ Real interpolation with logarithmic functors, J. Inequal. Appl. 7 (2002), no. 2, 187-269

[15] D. E. Edmunds and H. Triebel, Sharp Sobolev embeddings and related Hardy inequalities: the critical case, Math. Nachr. 207 (1999), 79-92.

[16] D. D. Haroske, Envelopes in function spaces - a first approach, Jenaer Schriften zur Mathematik und Informatik Math/Inf/16/01, 2001, pp. 1-72.

[17] A. Kufner, O. John, and S. Fučík, Function spaces, Noordhoff International Publishing, Leyden, 1977.

[18] B. Opic and A. Kufner, Hardy-type inequalities, Pitman Research Notes in Mathematics Series, vol. 219, Longman Scientific \& Technical, Harlow, 1990.

[19] B. Opic and L. Pick, On generalized Lorentz-Zygmund spaces, Math. Inequal. Appl. 2 (1999), no. $3,391-467$

[20] B. Opic and W. Trebels, Sharp embeddings of Bessel potential spaces with logarithmic smoothness, Math. Proc. Cambridge Philos. Soc. 134 (2003), no. 2, 347-384 
[21] J. Peetre, Espaces d'interpolation et théorème de Soboleff, Ann. Inst. Fourier (Grenoble) 16 (1966), no. 1, 279-317.

[22] H. Triebel, Sharp Sobolev embeddings and related Hardy inequalities: the sub-critical case, Math. Nachr. 208 (1999), 167-178.

[23] _ The structure of functions, Monographs in Mathematics, vol. 97, Birkhäuser Verlag, Basel, 2001.

[24] N. S. Trudinger, On imbeddings into Orlicz spaces and some applications, J. Math. Mech. 17 (1967), 473-483. 